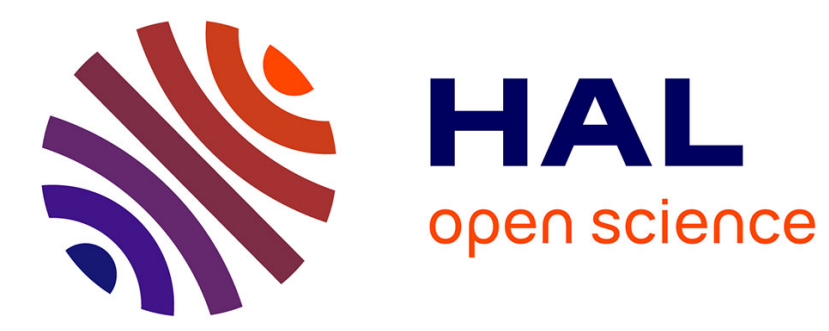

\title{
Asymmetric soft-hard colloidal mixtures: Osmotic effects, glassy states and rheology
}

Maria Consiglia Merola, Daniele Parisi, Domenico Truzzolillo, Dimitris

Vlassopoulos, Vishnu D. Deepak, Mario Gauthier

\section{To cite this version:}

Maria Consiglia Merola, Daniele Parisi, Domenico Truzzolillo, Dimitris Vlassopoulos, Vishnu D. Deepak, et al.. Asymmetric soft-hard colloidal mixtures: Osmotic effects, glassy states and rheology. Journal of Rheology, 2018, 62 (1), pp.63 - 79. 10.1122/1.5009192 . hal-01652734

\section{HAL Id: hal-01652734 \\ https://hal.science/hal-01652734}

Submitted on 8 Jun 2021

HAL is a multi-disciplinary open access archive for the deposit and dissemination of scientific research documents, whether they are published or not. The documents may come from teaching and research institutions in France or abroad, or from public or private research centers.
L'archive ouverte pluridisciplinaire HAL, est destinée au dépôt et à la diffusion de documents scientifiques de niveau recherche, publiés ou non, émanant des établissements d'enseignement et de recherche français ou étrangers, des laboratoires publics ou privés. 


\title{
Asymmetric soft-hard colloidal mixtures: osmotic effects, glassy states and rheology
}

\author{
Maria Consiglia Merola ${ }^{1,2}$, Daniele Parisi, Domenico Truzzolillo ${ }^{3}$, Dimitris Vlassopoulos \\ FORTH, Institute of Electronic Structure and Laser, and University of Crete, Department of Materials \\ Science and Technology, 70013 Heraklion, Crete, Greece. \\ Vishnu D. Deepak, Mario Gauthier \\ University of Waterloo, Department of Chemistry, Waterloo, ON N2L 3G1, Canada
}

\begin{abstract}
Whereas mixtures of colloids and non-adsorbing polymers have been studied in great detail in the last two decades, binary colloidal mixtures have not received much attention. Yet, fragmental evidence from asymmetric mixtures of hard spheres indicates a wide-ranging, complex behavior from liquid to crystal to single glass and to double glass, and respective rich rheology. Recently, we addressed the question of softness by investigating a mixture of soft and virtually hard colloidal spheres. We found an unprecedented wealth of states including repulsive single glass (RG), liquid, arrested phase separation (APS) and double glass (DG). This is a consequence of the coupling of softness and osmotic forces due to the hard component. We now report on the rheology of the different states with emphasis on the nonlinear response during start-up of stress at constant rate, its relaxation upon flow cessation, and large amplitude oscillatory shearing. Distinct features are identified, whereas comparison with single-colloid (soft or hard) glasses reveals some phenomenological universalities in yielding, residual stresses and periodic intra-cycle stress response. In brief, the DG exhibits much larger yield and residual stresses as compared to the RG and APS, whereas the yield strain is the same for all states. Two-step yielding is unambiguously evidenced for the APS whereas both yield stress and strain exhibit a weak dependence on Péclet number. Large amplitude oscillatory tests reveal large value of the intrinsic nonlinear parameters, reflecting the role of colloidal interactions. Moreover, RG exhibits intra-cycle stress

\footnotetext{
${ }^{1}$ merolamc@stanford.edu

${ }^{2}$ Present address: Department of Chemical Engineering, Stanford University, Stanford, CA 94305, USA

${ }^{3}$ Present address: Laboratoire Charles Coulomb (L2C), UMR 5221 CNRS - Université de Montpellier, Montpellier, France
} 
overshoots, a feature that characterizes most of the soft glassy materials formed by interpenetrable particles and that vanishes as hard (nearly impenetrable) colloids are added in the mixtures. These results demonstrate the sensitivity of linear and nonlinear rheology to colloidal state transitions and, more importantly, the power of entropic mixing as a means to tailor the flow properties, hence performance and handling of soft composites.

\section{INTRODUCTION}

Colloid-polymer mixtures serve as a paradigm for entropic manipulation of the flow properties of soft matter, while at the same time offering unique opportunities for addressing grand challenges in the field, especially that of the glass transition. Soft colloids, like star polymers, offer the further opportunity to tune the "softness" of mixtures by changing their molecular architecture and for this reason, their usage opens new perspectives for the formulation of novel soft materials in fields ranging from chemicals to foodstuff and medical applications. In this respect, star polymers serve as archetypes of tunable soft colloids: they are well-defined and allow linking the macroscopic properties to the internal particle microstructure and molecular characteristics. Indeed, during the last decades star polymers have been employed for several formulations or processes spanning from the synthesis of industrial thermoplastic elastomers [1] to drug and gene delivery, from tissue engineering [2], [3], to their usage as in-vivo therapeutic and diagnostic vehicles, interfacial stabilizers, and nanoreactors for catalysis [4]. The interest in using star polymers rises from their unique physico-chemical characteristics: low viscosity in dilute solutions, encapsulation capability, internal and peripheral functionality, and enhanced stimuli-responsiveness [3].

Even if the actual colloid-polymer (depletion) interactions were first considered in 1954 by Asakura and Oosawa [5], it is in the last 15 years that the field has evolved substantially [6], [7],[8],[9] especially with respect to the implications on the rheology of mixtures [10]. In a nutshell, mixtures of hard colloidal spheres and much smaller (about 1/10 in size) non-adsorbing polymers exhibit intriguing state behavior due to polymer-mediated depletion effects: At low colloid volume fractions, a liquid transforms into a gel upon increasing the concentration of polymer additives; at high colloid fractions in 
the repulsive glass $(\mathrm{RG})$ regime, the addition of polymers will melt the glass and yield an ergodic pocket of liquid (L), which however will give rise to a re-entrant attractive glass (AG) upon further increase in polymer concentration. Distinctive signatures of the $\mathrm{AG}$, as compared to the $\mathrm{RG}$, are a much larger (plateau) modulus and more complex yielding behavior (occurring in typically 2 steps instead of 1). These are attributed to the induced entropic particle bonding within the effective cages in the glassy state [11], [6], [10], [8], [12], [13].

Introducing softness via long-range soft potentials was found to impart both quantitative and qualitative differences in the vitrification of colloids [14], [15], [16], [17] and the behavior of colloidpolymer mixtures [16]. The key difference is the fact that the osmotic pressure exerted by the linear polymer additives may shrink the soft particles (causing a change in volume fraction) before depleting them. The main experimental system investigated systematically is the star polymer, which represents an idealized grafted, long hairy particle. This is due in part to the fact that it is relatively easy to change its softness and observe or estimate its osmotic compression [18], [19], [20]. Therefore, an experimental system well-understood at molecular level is used as model to study a complex problems of soft composites, hence it makes the methodology simple, sound and easy to follow or reproduce with similar systems [21], [22], [23]. The resulting state diagram is very rich [20], [24]. As for hard spheres, at large star fractions (above the glass transition), a viscoelastic liquid regime is detected upon increasing linear polymer concentration. A further addition of small linear chains eventually gives rise to a re-entrant solid state dominated by attractions: a gel whose dynamics is uniquely dictated by percolating shrunk stars. The presence of such a gel state is one of the characteristics of highly deformable colloids and differs largely from AG: while attractive glasses present both caging and bonding and they are characterized by a significantly larger storage modulus when compared to the original repulsive glass, on the other hand star-polymer gels are characterized by the absence of caging and much lower values of the moduli. Moreover, due to the range of the potential, the liquid pocket at intermediate volume fractions of linear polymer is larger than in the hard sphere case [21], [23], [22]. Related studies involve the structure and dynamics of mixtures of polymer grafted nanoparticles and linear polymers in good 
solvents [25] or mixtures of block copolymers and linear homopolymers in selective solvents [26], [27] and mixtures of microgels with different crosslinking density [28]. Adding polymers to microgel glasses was also found to induce melting and eventual re-entrance AG formation, with the extension of the ergodic region depending on the microstructure of the particles (e.g., the crosslinking density) [7], [12], [15], [29], the size ratio and the potentially changing solvent quality for the polymer. Similarly to star polymers, slightly crosslinked microgels or nanoemulsions were found to de-swell in the presence of small linear polymers[30], [31], [32], [33], [34], [35], [36]. Along these lines, studies with food protein systems, which are also soft, confirmed the qualitative universality of this behavior [37].

Whereas it is natural to consider the consequences of entropic mixing in different situations, as for example in binary colloidal mixtures, such studies have been rather limited. In fact, the morphology of binary asymmetric mixtures of hard spheres was studied with special emphasis on the existence of freezing transitions and the formation of superlattice structures, driven by the maximization of entropy as the system adopts its most efficient packing arrangement. It was actually found that for a given size ratio, highly asymmetric binary hard sphere mixtures may exhibit both crystalline and glassy regions of varying strength, depending on composition [38], [39], [40], [41], [42]. The latter can be single and double glasses, SG and DG, respectively, depending on whether one or both types of particles become kinetically trapped in amorphous structures. The role of softness has been addressed by systematically investigating mixtures of small and large particles of different softness, such as star polymers of different functionalities [43], [44], [45], [46]. Again, softness was responsible for the formation of different types of arrested states: ergodic mixtures, SG, DG and an asymmetric glass (AsG) where the effective large particle cages become anisotropic due to the osmotic pressure of the small particles. Along these lines, small microgels particles were used as depletants to large polystyrene hard sphere latexes [47], [48]; it was found that the induced gels were formed more easily than in mixtures of the same polystyrenes and linear polymer coils at a similar size ratio and that the different gel states had the same thermodynamic origins, irrespectively of differences in the detailed mechanism of the short-range 
attraction. More recently, binary mixtures of self-suspended grafted nanoparticles (silica particles with covalently bonded small polyethylene glycol chains, in the absence of solvent) were investigated [49].These systems have been proposed as a paradigm of solvent-free colloids [50], [51]. It was found that adding larger particles to a glassy suspension of smaller particles led to its softening, and in the limit of large size disparities, to its complete fluidization. This was shown to reflect a speed-up of decorrelation dynamics of all the particles in the suspension and a reduction in the energy dissipated at the yielding transition. We note, for completeness, that mixtures involving block copolymer micelles with frozen and "living" cores and short arms were studied as well, and it was found that a balance between crystallization and vitrification is observed as the number of arms is varied [52]. It is also important to mention that the interplay of RG and AG as well as gelation, can be also monitored via enthalpic and charge interactions as well, by using temperature-sensitive and/or charged colloidal systems [53], [54], [55], [56], [57], [58]. However, this route will be not considered in this work which focuses on athermal systems.

The above-mentioned ability to obtain different morphologies by entropic mixing, i.e., by combining soft and hard interactions, has opened the door for tailoring the flow properties of soft composites by molecular design of appropriate systems. Consequently, considering mixtures of small hard spheres and large star polymers has emerged as a natural challenge to further enhance these capabilities and explore new properties via experiments, simulations and theory [59], [60], [61]. In this spirit, we have recently reported on the state diagram of such mixtures [61], [45], [62] that is here sketched in Figure 1. 


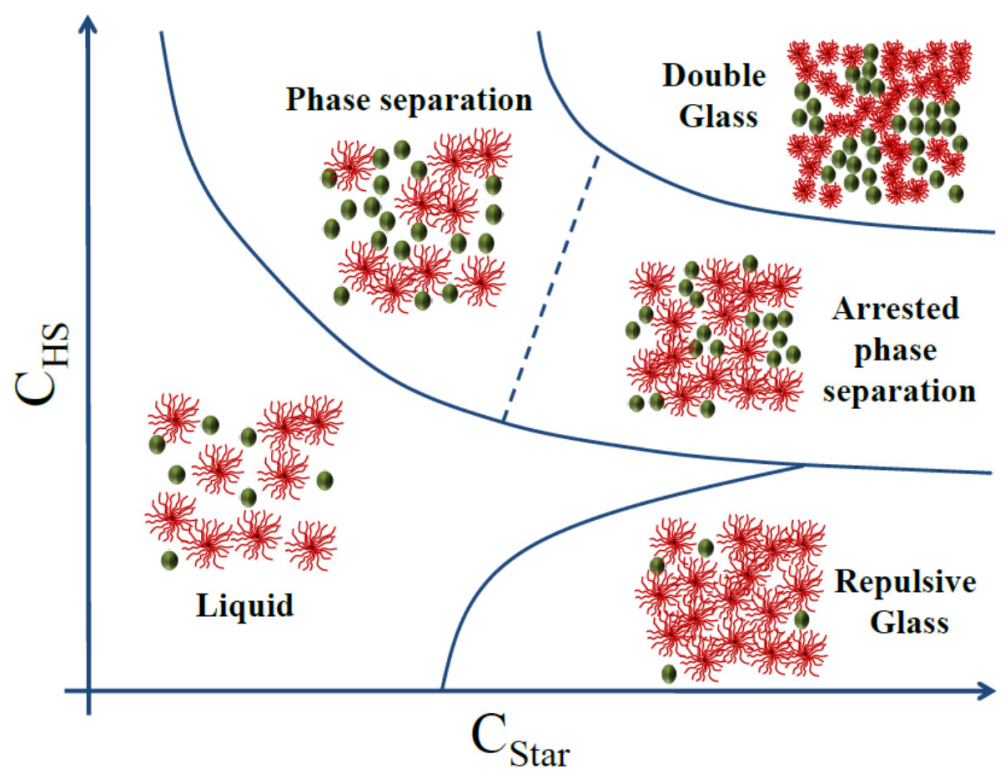

Figure 1: Schematic of the state diagram of hard-sphere/star mixtures.

We found that at low polymer star volume fractions the colloidal liquid phase separates upon adding hard spheres, whereas at higher volume fractions, the RG of the soft star progressively weakens, it gives rise to a viscoelastic liquid and eventually to re-entrant arrested phase separation and DG upon continuous addition of hard spheres. These findings are based on experimental evidence from linear viscoelasticity and dynamic light scattering, as well as modelling using MCT, supported by MD simulations. An important open question is the rheological implications of this very rich morphology, in particular regarding nonlinear shear rheology which has been barely investigated with soft colloidal mixtures. This represents the subject of the present work.

We present a comparative rheological investigation of different states of asymmetric binary softhard colloid mixtures. Using the same system studied before [61], [62], we extend our earlier studies and report on the transient nonlinear rheology (evolution and relaxation of stress under constant shear, response to large amplitude oscillatory shear deformation) of RG, APS and DG states. We discuss the phenomenological link of the rheological properties to morphology and compare simple repulsive glasses from hard spheres, microgels and stars. The results can serve as a guide for further advancing 
our theoretical understanding and hopefully will stimulate more experimental research into this exciting field.

\section{EXPERIMENTAL SYSTEMS AND METHODS}

\section{II.1.Materials:}

We used the experimental system of references [61] and [62]. It consists of two different multiarm 1,4polybutadiene (PBD) stars, as soft and hard sphere respectively, by taking advantage of the well-known fact that their pair interaction potential depends on star functionality, $f$. In this way, we ensured that the mixture is purely entropic. On the other hand, the hard star is softer than experimental hard sphere systems (PMMA latex or silica particles), however this does not affect the message of the work, as discussed below. Details about the synthesis of the stars can be found elsewhere [63], [62]. Their molecular characteristics are listed in the table 1 below.

The size exclusion chromatography (SEC) traces of both the soft and hard sphere (HS)-like star polymers are shown in Figures 2 (A,B) and they are compared with the traces of the linear chains which are grafted to the cores. The SEC traces were obtained using a Viscotek GPC max unit equipped with a VE 2001 GPC Solvent/sample Module, a Viscotek triple detector array equipped with refractive index, viscosity, and dual-angle light scattering $\left(7^{\circ}\right.$ and $\left.90^{\circ} \lambda=670 \mathrm{~nm}\right)$ detectors (allowing to determine the weight-average molar mass by means of a simplified Zimm-like plot), and an external Viscotek UV 2600 detector (Malvern, UK).The SEC traces shown in Figures 2 (panels A and B) demonstrate that both soft and HS-like star polymer samples have virtually no free chains. Nonetheless, a small shoulder in the molar mass distribution of the HS-like stars $(f=1109)$ can be detected with high hydrodynamic volume. We fitted the mass distribution with two Lorenz functions and calculated the relative heights of the distributions to estimate the fraction of high molecular weight contaminant. We found that the mass fraction of contaminant is 0.036 . We performed the same analysis of the SEC traces also in the case of the soft stars $(f=214)$ to estimate the fraction of low molecular weight contaminant that is possibly due, in this case, to the presence of stars with smaller functionality. From the relative height of the 
distributions (Figure 2) we found that the molar fraction of contaminant is 0:12. SEC analysis served to determine also the polydispersity index $\left(\mathrm{PDI}=M_{w} / M_{n}\right)$ values for the branched polymers: $\mathrm{PDI}=1.07$ for the soft stars and PDI=1.11 for the hard-sphere-like stars.

A useful, yet approximate way to characterize the softness of the stars is by means of the Daoud Cotton model[64]. In particular, we can use a softness parameter $S P=R_{c} / R_{s}$, where $R_{s}$ and $R_{c}$ are the radii of the whole star and the core, respectively. In the context of the Daoud-Cotton model, for the soft starwe are in the swollen regime $N_{a}>f^{1 / 2} v^{-2}$ (where $N_{a}$ is the degree of polymerization of the arm and $v$ the excluded volume of the monomer) and the effective core represents the un-swollen regime, whereas its hard counterpart is in the $\operatorname{limit} N_{a} \approx f^{1 / 2}$ without swollen region. The respective softness values are roughly $S P=f^{3 / 10} N_{a}^{-3 / 5} \approx 0.07$ and $>1$ for the soft and the hard star, respectively (see Table 1). A value for SP higher than 1 is just a sign of the ultra-compact nature of our HS-like stars and is a common feature for stars with $f>1000$ and short arms $\left(N_{a}<30\right)$, whose description necessitates to go beyond the Daoud-Cotton scaling model. 


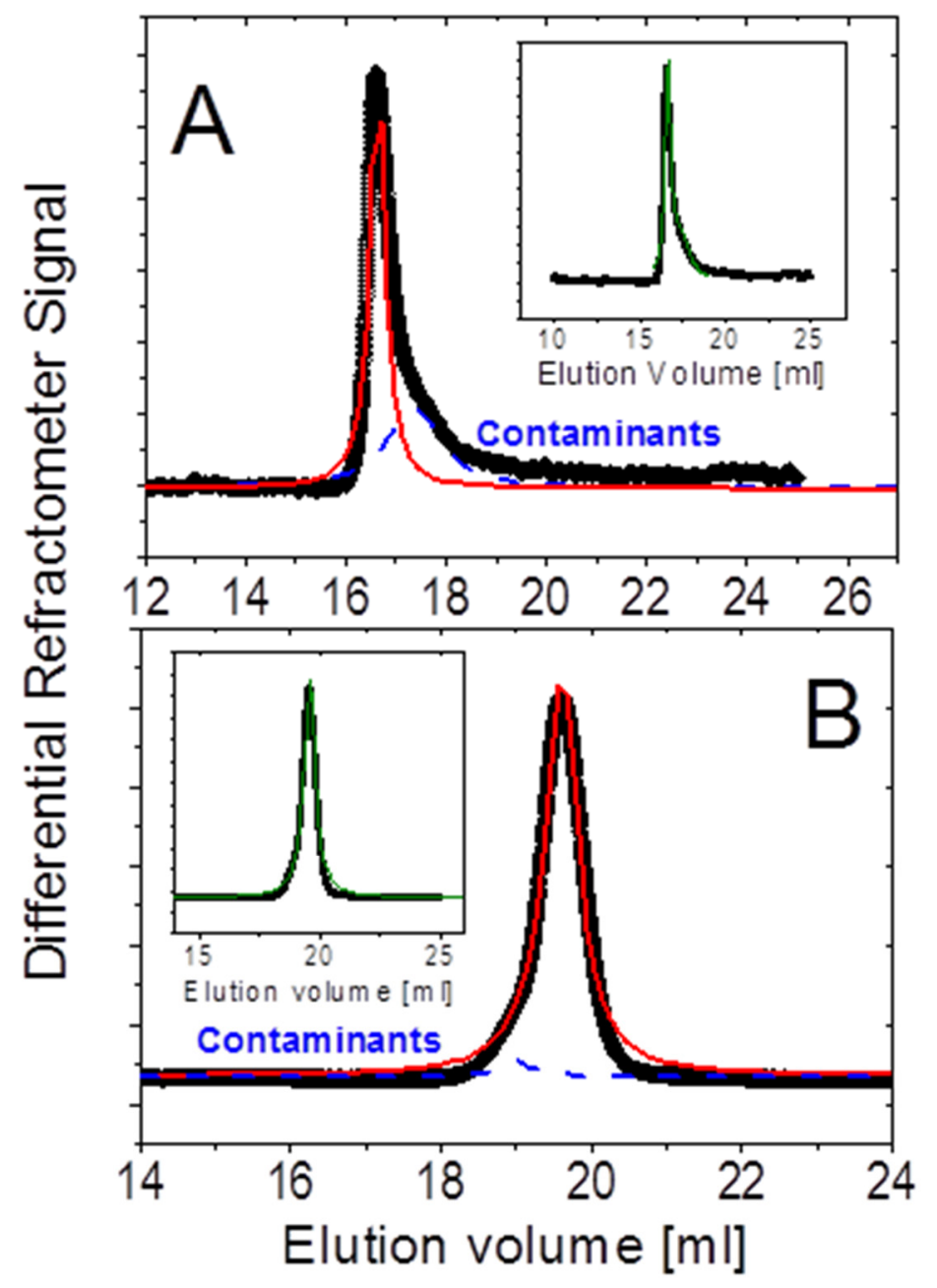

Figure 2: Size exclusion chromatography trace of the soft stars (panel A) HS-like stars (panel B), along with their fits with two convoluted Lorenz functions. Respective insets: The resulting total fit is shown by the solid line (red curve online).

TABLE 1. Molecular characteristics of the star polymers

\begin{tabular}{ccccccccc}
\hline \hline Sample & $\mathrm{f}$ & $\begin{array}{c}\text { Marm } \\
(\mathrm{g} / \mathrm{mol})^{\mathrm{a}}\end{array}$ & $\begin{array}{c}\mathrm{R}_{\mathrm{h}} \\
(\mathrm{nm})^{\mathrm{b}}\end{array}$ & $\begin{array}{c}\mathrm{c}^{*} \\
(\mathrm{mg} / \mathrm{ml})\end{array}$ & $\mathrm{SP}$ & $\Phi_{\mathrm{eff}, \mathrm{g}=\mathrm{cg} / \mathrm{c}^{*}}$ & Mw/Mn (PDI) & $\begin{array}{c}\text { Mw } \\
(\mathrm{g} / \mathrm{mol})\end{array}$ \\
\hline Hard & 1109 & 1270 & 11.5 & 375.1 & $>1$ & 0.75 & 1.11 & $1.6 \times 10^{6}$ \\
Soft & 214 & 67000 & 45 & 62.3 & 0.07 & 1.61 & 1.07 & $1.5 \times 10^{7}$ \\
\hline \hline
\end{tabular}

${ }^{\text {a }}$ Average molecular weight of one single arm.

${ }^{\mathrm{b}}$ Hydrodynamic radius estimated from the diffusion coefficient (DLS) at $20^{\circ} \mathrm{C}, c^{*}=\left[(4 \pi / 3) R_{h}^{3}\right]^{-1}$ $\left(f M\right.$ arm $\left./ N_{A}\right)$. 


\section{II.2. Rheological protocols:}

The rheological state of the samples was investigated via rheometric measurements, carried out with a sensitive strain-controlled rheometer (ARES-HR 100FRTN1 from TA USA). All measurements were performed with a cone-and-plate geometry (home-made stainless steel cone of $8 \mathrm{~mm}$ diameter and 0.166 rad cone angle). The temperature was set at $20.00 \pm 0.01{ }^{\circ} \mathrm{C}$ by means of a Peltier plate with a recirculating water/ethylene glycol bath. The samples were loaded on the rheometer and, after applying a well-defined pre-shear protocol which ensured that they were properly rejuvenated before testing [65], their linear dynamic response was measured at different times. This means that the rejuvenated samples were in the liquid state at the end of pre-shearing (i.e., the stress nearly dropped to zero), and from then on it was possible to account for aging, and hence establish conditions for reproducible and virtually time-independent measurements. We emphasize the importance to establish such a protocol when investigating metastable systems, and follow it carefully in all experiments (tests, samples) in order to obtain consistent results that could be interpreted in light of this. The exact experimental protocol consisted of the following steps: (i) The sample underwent a dynamic strain amplitude sweep (DSS) at a fixed frequency $(1 \mathrm{rad} / \mathrm{s})$ in order to determine the range of strains corresponding to liquid-like response. (ii) Then a dynamic time sweep (DTS) at a chosen strain $(200 \%)$ and $\omega=1 \mathrm{rad} / \mathrm{s}$ was performed in order to fully rejuvenate the liquefied sample, as judged from the time-independent apparent moduli (this lasted 300 s). (iii) After a chosen rest time, a dynamic time sweep was performed in the linear regime, in order to follow the time evolution of moduli until nearly constant values were reached, corresponding to the aged sample. Typically, the time scale for this was about 1 day. (iv), Small-amplitude oscillatory shear (SAOS) frequency sweep tests (DFS) were subsequently carried out in a frequency range 0.1-100 $\mathrm{rad} / \mathrm{s}$. Such protocol was preliminarily used to determine the concentrations $c_{g}$ corresponding to the rheological glass transitions for both the stars (Figure 4). We obtained $\mathrm{c}_{\mathrm{g}}=(1.61 \pm 0.01) \mathrm{c}^{*}$ for soft stars and $\mathrm{c}_{\mathrm{g}}=(0.75 \pm 0.05) \mathrm{c}^{*}$ for HS-like stars. It is worth noting that for liquid samples at concentrations close to $c_{\mathrm{g}}$, the crossover of the two moduli does not coincide with the onset of terminal relaxation $\left(\mathrm{G}^{\prime} \sim \omega^{2}\right.$, $\left.\mathrm{G}^{\prime \prime} \sim \omega^{1}\right)$; this reflects the large distribution of relaxation times that characterizes crowded star polymers 
$[34,35,66]$, [67]. (v) The response to large amplitude oscillatory shear(LAOS) of all the aged samples was investigated via dynamic strain sweep tests, carried out at fixed frequencies ( $1 \mathrm{rad} / \mathrm{s}$ and $10 \mathrm{rad} / \mathrm{s}$ ) with strain amplitudes covering the range from linear $\left(\gamma_{0} \leq 1 \%\right)$ to strongly nonlinear $\left(\gamma_{0} \approx 300 \%\right)$ regimes. The whole protocol is represented schematically in Figure 3 below.

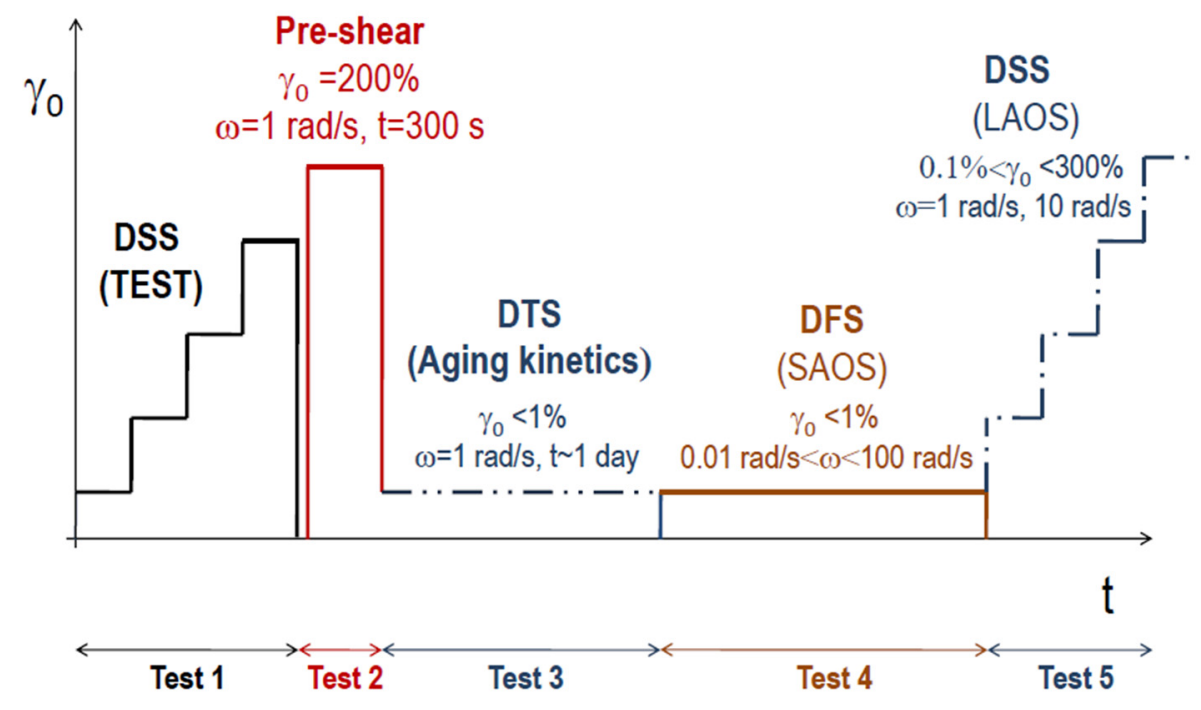

Figure 3: Experimental protocol used to determine the rheological state of the mixtures and their nonlinear response ( $\gamma_{0}$ and $\omega$ are respectively the strain amplitude and the frequency of the oscillatory tests). 


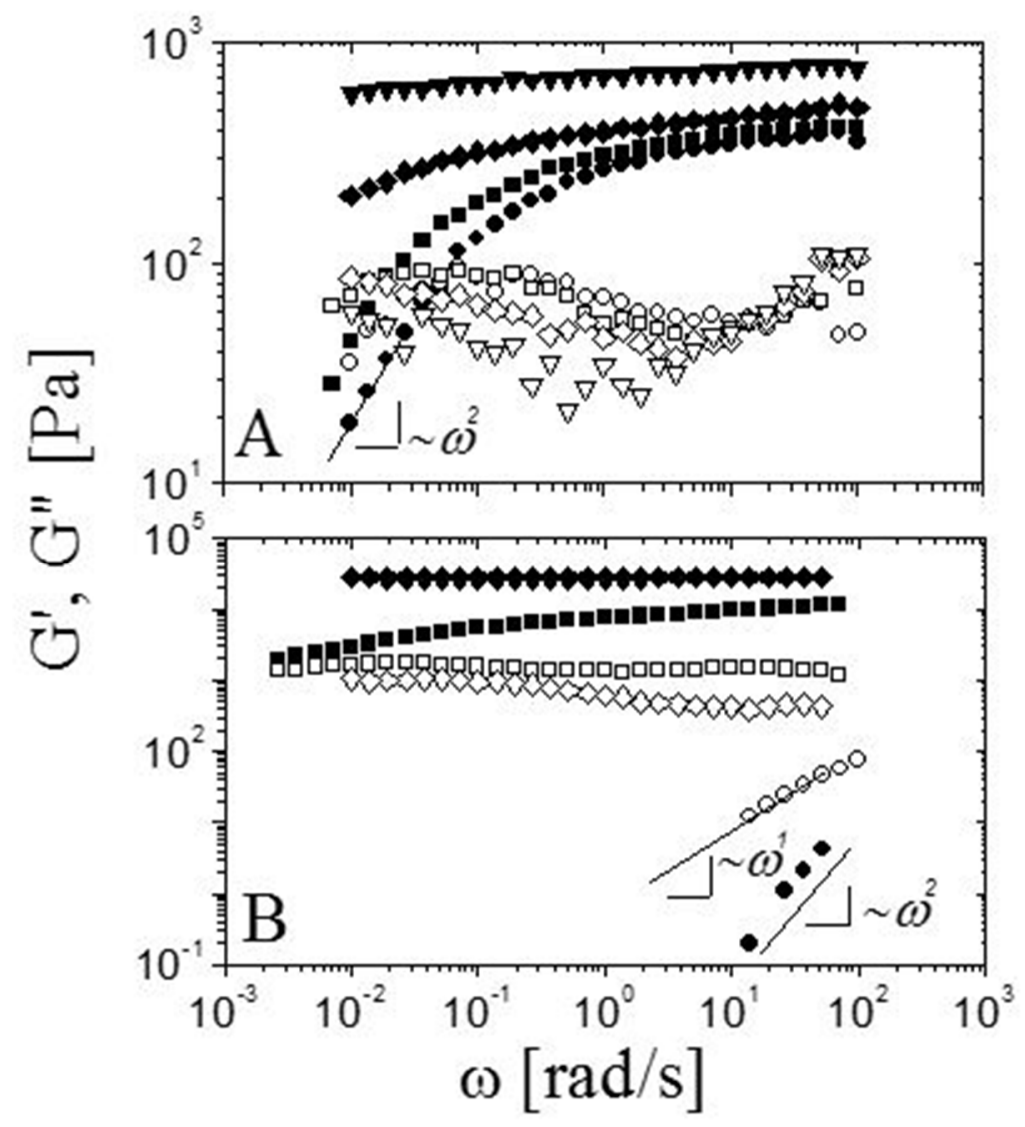

Figure 4: Linear viscoelastic spectra for soft-star or simply star (Panel A) and hard star or simply hardstar (Panel B) across the rheological glass transition. Panel A: G' (full symbols) and G"(open symbols) for soft-stars at $c=1.5 c^{*}$ (circles), $c=1.6 c^{*}$ (squares), $c=1.625 C^{*}$ (lozenges), $c=1.65 c^{*}$ (down-triangles). Panel B: G' (solid symbols) and G"(open symbols) for hard-stars at $c=0.7 c^{*}$ (circles), $c=0.8 c^{*}$ (squares), $c=0.9 c *$ (lozenges).

Step-rate and stress relaxation measurements were performed for the three different solid-like states found by varying the content of HS-like stars: repulsive glass (RG), arrested phase separated mixtures (APS) and double glass (DG). Different shear rates, from $0.001 \mathrm{~s}^{-1}$ to $0.5 \mathrm{~s}^{-1}$, were applied after sample rejuvenation (consisting in a dynamic time sweep at $\gamma_{0}=200 \%$ and $\omega=1 \mathrm{rad} / \mathrm{s}$ for $100 \mathrm{~s}$ ) and a dynamic time sweep in the linear regime (typically at $\gamma_{0}=1 \%$ and $\omega=1 \mathrm{rad} / \mathrm{s}$ for $300 \mathrm{~s}$ ) to ascertain the quasi-stationary values of the moduli. The experimental time for the step-rate tests was set to reach the same maximum accumulated strain $(\gamma=50 \%)$ for all the mixtures. Finally, after each step-rate test, the stress relaxation was monitored for 200 seconds. 


\section{RESULTS AND DISCUSSION}

\section{III.1. Linear viscoelasticity}

Selected linear viscoelastic spectra are shown in Figure 5A, where storage the $\left(G^{\prime}\right)$ and loss $\left(G^{\prime \prime}\right)$ moduli are plotted at varying HS-like star concentration $\mathrm{c}_{\mathrm{HS}}$ and fixed concentration of soft stars $\left(\mathrm{c}_{\mathrm{s}}=1.625 \mathrm{c}_{\mathrm{s}}^{*}>\mathrm{cg}_{\mathrm{g}}\right)$. The system, initially in a repulsive glassy state in the absence of HS-like star additives, reaches an ergodic liquid-like state for $\mathrm{c}_{\mathrm{HS}} \geq 0.2 \% \mathrm{wt}$. As the concentration of HS-like stars is increased further, the mixture re-solidifies ( $\mathrm{cHs}>3 \% \mathrm{wt}$ ) and transforms into an arrested phase-separated state [61],characterized by a storage modulus similar to that of the initial repulsive glass ( $\mathrm{cHs}=0 \% \mathrm{wt}$ ), hence suggesting that the re-entrant state is not an attractive glass (Figures 5 and 6). Indeed attractive glasses are characterized by larger values of the storage modulus when compared to repulsive glasses, due to the presence of both caging and attractive forces. On the contrary, in the present case, the glass line hits the demixing (spinodal) line of the mixture and the star-rich phase gets arrested into a new solid state that is rheologically equivalent to the RG state [61].The two states (RG and APS) exhibit quantitative differences in their dynamics: For instance, the time associated with the slowest intra-cage $\beta$-relaxation mode, estimated as the inverse frequency at the minimum of $\mathrm{G}^{\prime \prime}(\omega), \omega_{\beta}=1 / \tau_{\beta}$, exceeds $5 \mathrm{~s}$ for APS states (see Figure 5) and cannot be detected precisely due to the large dispersion of the data. This is not the case for the initial repulsive glass where $\tau_{\beta}$ does not sensibly depend on HS-like star concentration (Figure 4B) and can be determined accurately, in the range $0.1 \mathrm{~s}<\tau_{\beta}<0.5 \mathrm{~s}$.

As the concentration continues to increase, the mixtures reach the DG state with a slower intra-cage dynamics (Figure 5B). In general, the increase of $\tau_{\beta}$ with particle concentration is attributed to the reduced free volume due to crowding [68]. Furthermore, in the DG state both depletants and depleted particles freeze, the elastic modulus increases sharply (Figure 6) while the soft stars shrink due to the osmotic pressure exerted by the HS-like stars [62]. Indeed, taking into account the mean-field pressure exerted by the HS-like stars [62] for $\mathrm{cHS}_{\mathrm{H}}>40 \% \mathrm{wt}$, soft stars become dramatically squeezed and assume 
a collapsed configuration akin to that observed under poor solvency conditions. In the present case, the soft star radius can drop continuously up to the limit of star collapse $R_{c} \approx b\left(f N_{a}\right)^{1 / 3} \approx 16$ nm (here $b=0.25$ $\mathrm{nm}$ is the monomer radius [69] and $\mathrm{N}_{\mathrm{a}}=1240$ is the degree of polymerization of one arm). The above estimation represents a lower bound for the real size of the collapsed soft star, i.e., the size of a spherical collection of jammed monomers. Accordingly, the shrunk stars can also be thought of as hard spheres that are slightly larger than the original colloids. Therefore, under these conditions the system is very similar to a slightly asymmetric binary hard sphere mixture as we have already pointed out in [62].

A recapitulatory scenario including the sequence of states of the mixtures is provided in Figure 6. At low colloid density, a repulsive-glass region is characterized by non-monotonic dependence of the plateau modulus Gp of the mixtures on HS-like star concentration due to the competition between reinforcement and depletion. Initially, the addition of hard spheres has a strong reinforcing effect on the star suspension, leading to an increase of $\mathrm{G}_{\mathrm{p}}$ by a factor of $56 \%$. Whereas this may resemble qualitatively to Guth-Gold type of behavior [70] (which would indicate a reinforcement of only $0.2 \%$ ), it is much stronger, due to the large fraction of the initial glassy suspension. However, with further HSlike star addition, depletion dominates and the mixture softens while it remains glassy. Once the mixtures enter in the basin of ergodic states, the characteristic time of the liquid (extracted from the terminal crossover of the moduli, i.e., $\left.\tau_{\mathrm{r}}=1 / \omega_{\text {crossover }}\right)$ varies over three decades; it first decreases with cHS, then goes through a minimum and finally increases as the re-entrance is approached. This is a generic behavior for an ergodic phase between two glassy states [61], [62], [71], [49]. The re-entrant solid-like state has been discussed and identified as an arrested phase separation [61], [62] whose rheology (dynamics) is dominated by that of soft stars. However, as mentioned before, when the concentration of hard stars increases and approaches $\mathrm{c}_{\mathrm{g}}{ }^{\mathrm{HS}}$, the plateau modulus of the mixtures increases sharply up to values slightly above those observed for (the same) pure HS-like stars at $\mathrm{c}_{\mathrm{g}}\left(\mathrm{G}_{\mathrm{p}} \approx 10^{4} \mathrm{~Pa}\right)$ and then increases as $\mathrm{G}_{\mathrm{p}} \sim \mathrm{cHS}^{\alpha}$ with $\alpha=2.0 \pm 0.2$. Such a scaling reflects the "hybrid" structure of our HSlike star polymers. Indeed, although such concentration dependence of the storage modulus is much 
weaker than that previously reported for glassy hard sphere fluids $\left(\mathrm{G}_{\mathrm{p}} \sim \mathrm{c}_{\mathrm{HS}}{ }^{50}\right)$ [72], [73], it also differs considerably from that found for densely grafted particles or other multiarm star polymers and should reflect the high functionality and compactness of the stars in the DG regime. Typically, for stars with $\mathrm{f} \approx 400$ a much weaker dependence $\left(\mathrm{G}_{\mathrm{p}} \sim \mathrm{c}_{\mathrm{star}}{ }^{1}\right)$ was observed [74], [19]. Hence, we suggest that for highly crowded stars, with more than 1000 arms, particle compactness along with steric repulsion between cores make stars akin to hard spheres and affect the collective dynamics of the suspensions.
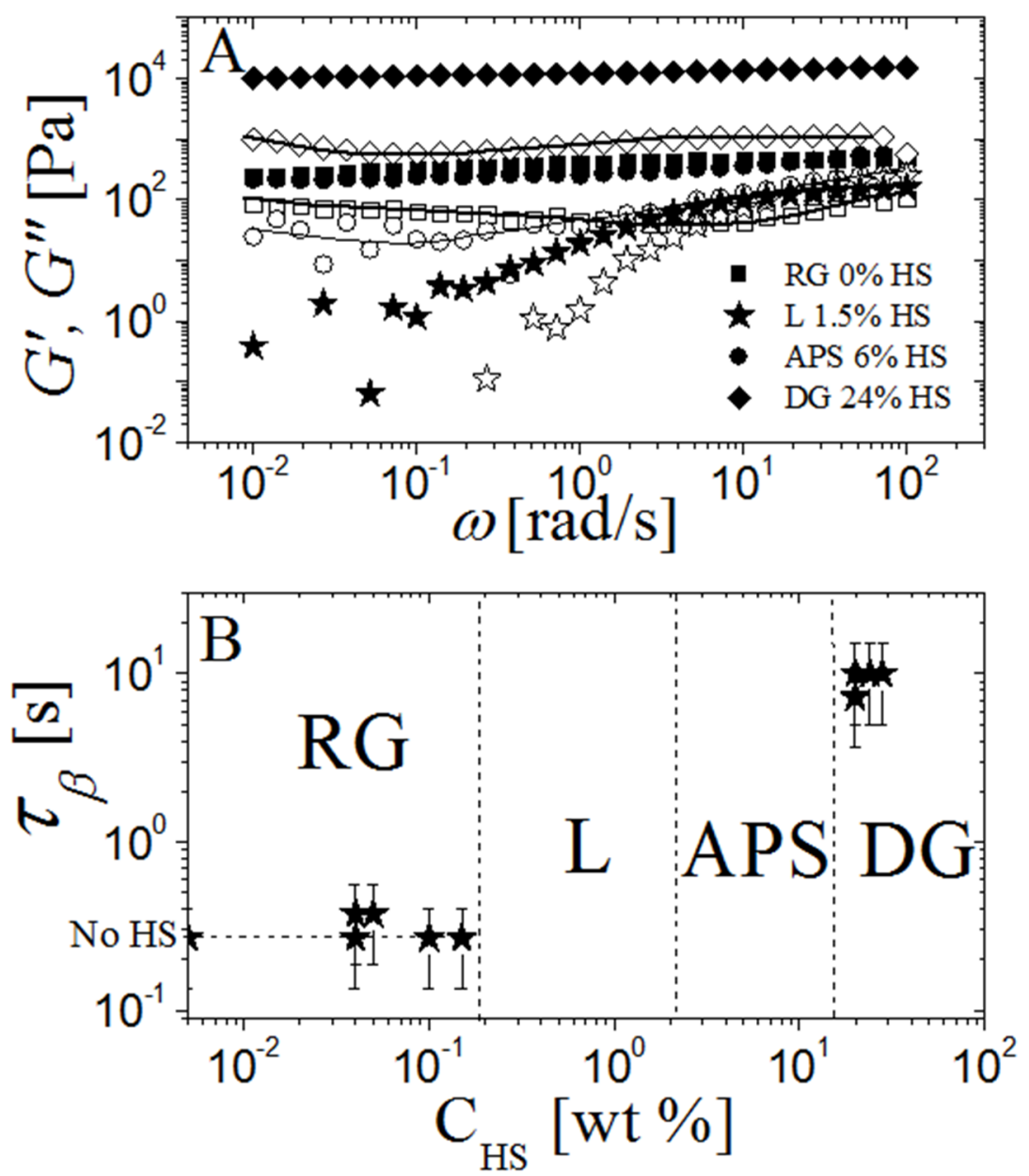

Figure 5: Linear viscoelastic (LVE) spectra of Star/HS mixtures at different HS concentrations (Panel A) and slowest $\beta$-relaxation time in function of HS concentration (Panel B). In Panel A the lines are drawn to guide the eye. In Panel B vertical dashed lines separate the different states of the mixtures and are located according to the criteria established by means of linear and nonlinear rheology: RG-L (LAPS) boundary is determined by looking at the onset (disappearance) of the crossover between $G^{\prime}$ and $\mathrm{G}^{\prime \prime}$ in the frequency range $10^{-2}<\omega<10^{2} \mathrm{rad} / \mathrm{s}$; the APS-DG boundary is drawn where the plateau modulus and the yield stress of the mixtures abruptly jump and change slope when plotted vs chs as explained in the text. The dotted line in the RG region is drawn to guide the eye. 


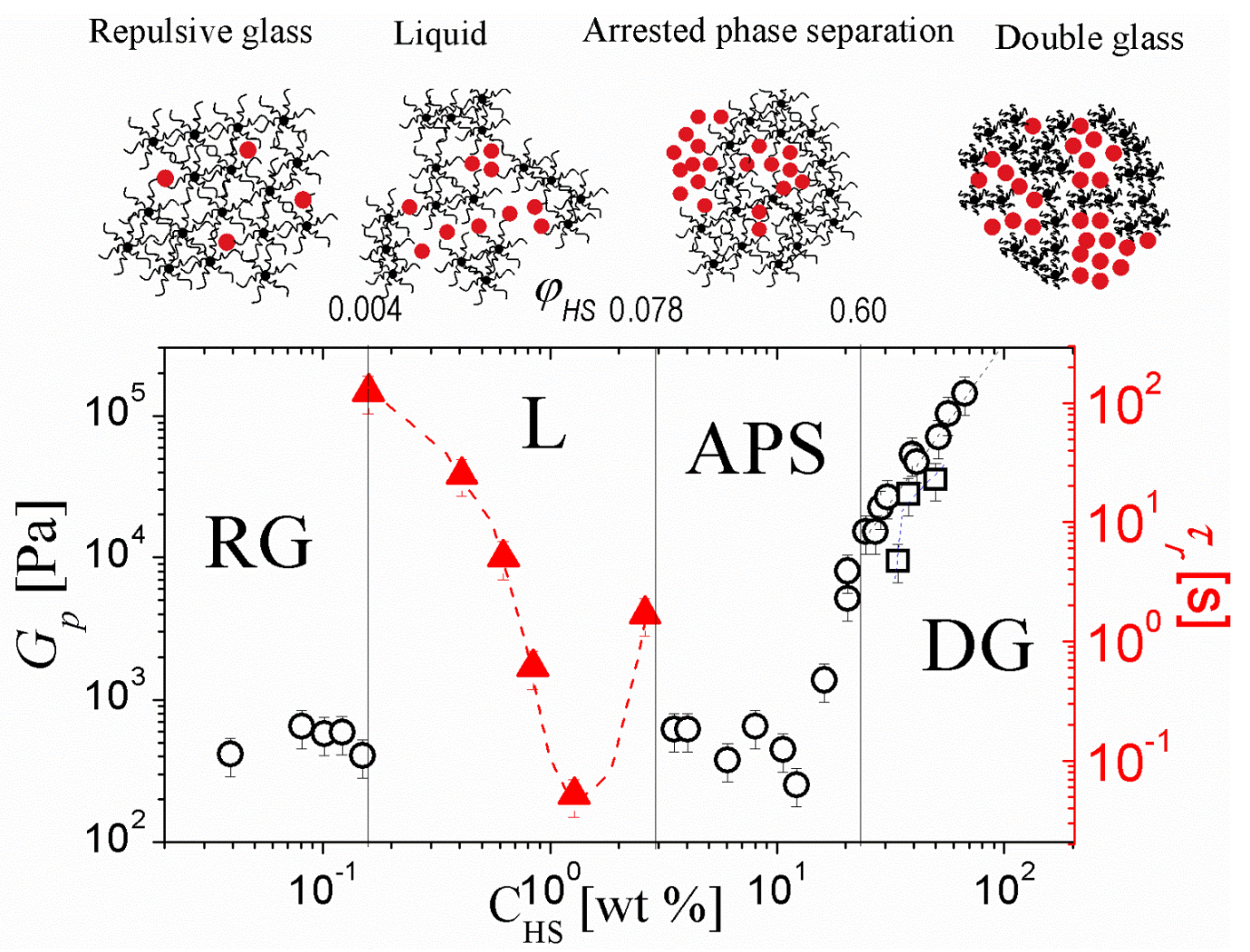

Figure 6: Plateau modulus for dynamically arrested mixtures (circles) and relaxation time (right axis) for liquid systems (red full triangles) in function of HS concentration across the different states sketched by the cartoons. The plateau modulus for three pure HS suspensions is also reported (squares).

\section{III.2. Strain amplitude sweeps and yielding}

The response of the mixture to an imposed Large Amplitude Oscillatory Shear (LAOS) strain and the yielding transition were examined with dynamic strain sweeps at different frequencies $(1 \mathrm{rad} / \mathrm{s}$ and 10 $\mathrm{rad} / \mathrm{s})$. Figure 7 shows representative data for the first harmonic of $\mathrm{G}^{\prime}\left(\gamma_{0}\right)$ and $\mathrm{G}^{\prime \prime}\left(\gamma_{0}\right)$ as a function of the strain amplitude for the different solid-like states (RG, APS, DG). As expected, for small amplitudes both moduli are insensitive to the amplitude of the applied strain: the sample obeys linear viscoelastic response. At larger strain amplitudes the onset of nonlinearity is marked by an increase of G", which eventually reaches a maximum before decreasing according to a power-law. At the same time, $\mathrm{G}^{\prime}$ decreases with strain smoothly and eventually follows a power-law with exponent almost double compared to that of G" as reported for different jammed systems [72], [75], [49], [71], [76]. This is not further discussed in this work. In this regime, the material begins to yield and switches from solid-like to liquid-like. While a single yield point can be identified in the RG state (Figure 7A1, B1) for both 
frequencies investigated, the re-entrant state (APS) exhibits a more complex yielding behavior: a twostep process (Figure 7 B2) characterizes the yielding transition and appears only at $10 \mathrm{rad} / \mathrm{s}$ in a small range of hard star concentrations $6 \% \mathrm{wt} \leq \mathrm{c}_{\mathrm{HS}} \leq 12 \%$ wt. For APS one can easily envisage two length scales, the initial cage and that of the colloid-rich phase that is being arrested.

At higher HS-like star concentrations $\left(\mathrm{cHS}_{\mathrm{HS}}>12 \% \mathrm{wt}\right.$ ), the single yielding behavior is recovered along with an increase in the modulus reflecting the loss of bonds within the mixture and the passage to a new glassy state (DG) dominated by caging. From the dynamic strain sweeps we extract the yield strain (Figure 8) and the yield stress (Figure 9) as the values obtained at the crossover point between $\mathrm{G}^{\prime}$ and G". In the case of mixtures showing two yield processes, the second yield point has been identified as the one corresponding to the maximum of G" appearing at high strains (see arrows in Figure 7-B2). For the mixtures characterized by a single yield process, $\gamma_{\mathrm{y}}$ does not exhibit any appreciable dependence on hard star concentration $\left(\gamma_{\mathrm{y}} \sim \mathrm{cHS}^{0}\right)$ or frequency: we obtain $\gamma_{\mathrm{y}} \approx 10 \%$, which falls in the typical range of values reported for colloidal suspensions [10], [77], [72], [73], [74]. Interestingly, the two yield strains appearing in the APS state at $10 \mathrm{rad} / \mathrm{s}$ are appreciably different from those characterizing the single yield point of the single (RG) and double (DG) glass states, suggesting that the very processes driving the yield of the suspensions in the APS state differ from those driving the yield of solid repulsive states [42], [49], [77]. Indeed, multiple yield processes are thought of as reflecting multiple constraining length scales (in our case dictated by bonds and cages) which occur in systems where interactions lead to additional length scale such as due to clustering in binary colloidal mixtures [77], [49], [42], attractive glasses [10], [71] or arrested phase separating systems alike. Given the yield strain behavior, the yield stress $\sigma_{\mathrm{y}}\left(=\mathrm{G}_{\mathrm{p}} \gamma_{\mathrm{y}}\right)$ expectedly follows the same qualitative trend observed for the plateau modulus $\mathrm{G}_{\mathrm{p}}$ (Figure 9). In particular, as already discussed, at low hard star fraction in the RG state the mixtures are characterized by non-monotonic dependence of the yield stress $\sigma_{y}$ on HS-like star concentration. Interestingly, the two solid-like states beyond the ergodic region (APS and DG) are characterized by two different trends of $\sigma_{\mathrm{y}}$ as function of $\mathrm{c}_{\mathrm{HS}}$. It exhibits a mild (albeit clear) dependence 
on $\mathrm{c}_{\mathrm{HS}}$ in the APS state and does not show any appreciable dependence on frequency. On the other hand, for samples characterized by two distinct yield points (see APS states at $\mathrm{cHS}_{\mathrm{HS}}=6 \% \mathrm{wt}$ and $\mathrm{cHS}=12 \% \mathrm{wt}$ at $10 \mathrm{rad} / \mathrm{s}$ ), a first yield process corresponding to the crossover between $G^{\prime}$ and $G^{\prime \prime}$ that reflects the cohesive failure of the glassy material, is marked by a yield stress equal to that measured at $1 \mathrm{rad} / \mathrm{s}$ for the same samples and is much lower than those obtained for all the other samples in this concentration range. We attribute such behavior to the nature of the dynamical arrest of the mixtures with entropic attraction due to depletion, and in particular the occurrence of bond breaking in this range of cHS.
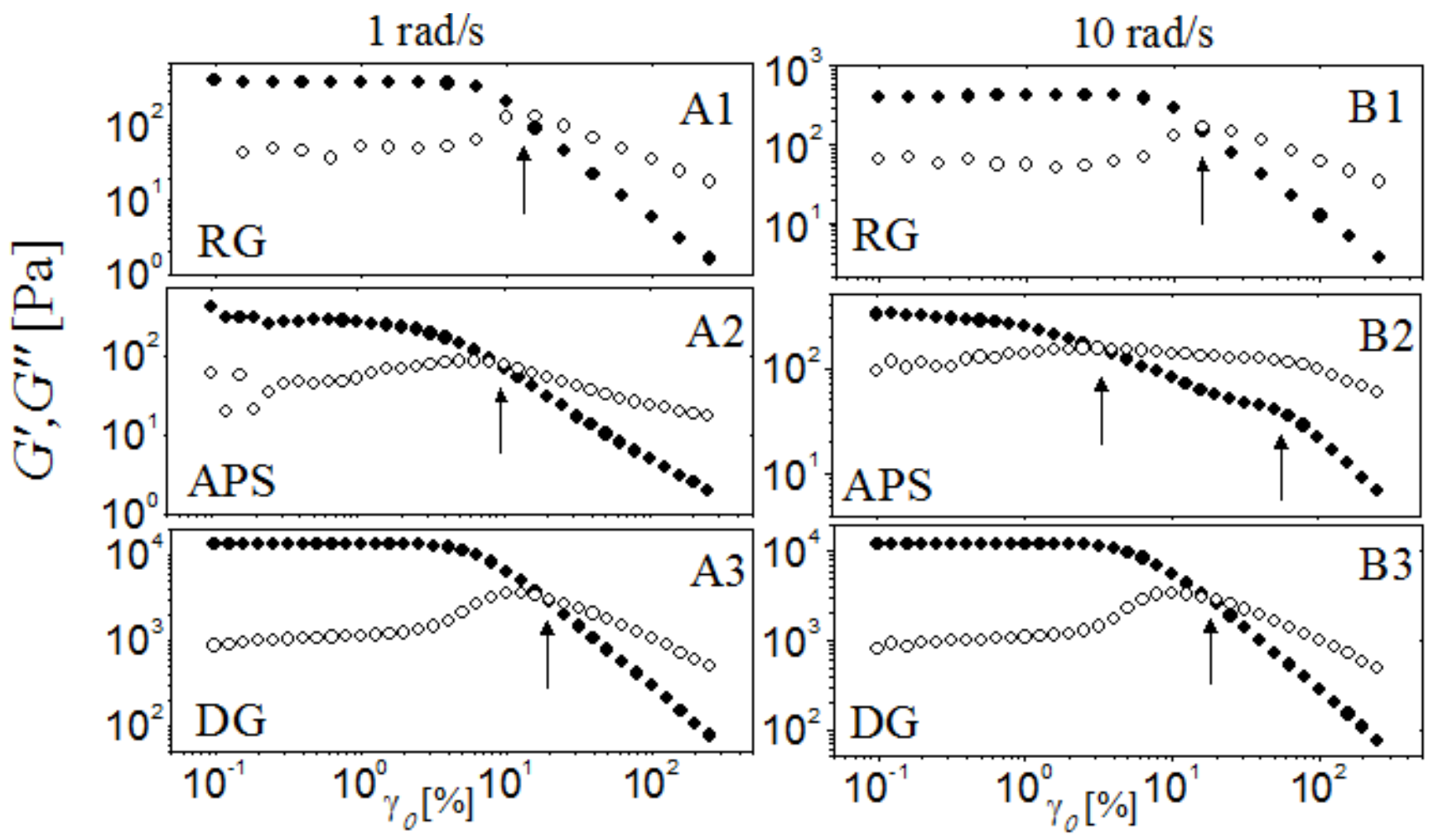

Figure 7: Dynamic strain sweeps at $1 \mathrm{rad} / \mathrm{s}$ and $10 \mathrm{rad} / \mathrm{s}$ for selected samples representative of the three solid states: Repulsive glass (Panels A1,B1 - chs=0 \% wt), Arrested Phase Separation (Panels A2,B2 $\mathrm{cHS}_{\mathrm{HS}}=6 \% \mathrm{wt}$ ), Double Glass (Panels A3,B3 - $\mathrm{c}_{\mathrm{HS}}=24 \% \mathrm{wt}$ ). Solid symbols refer to $\mathrm{G}$ ' and open symbols to $\mathrm{G}^{\prime}$. 


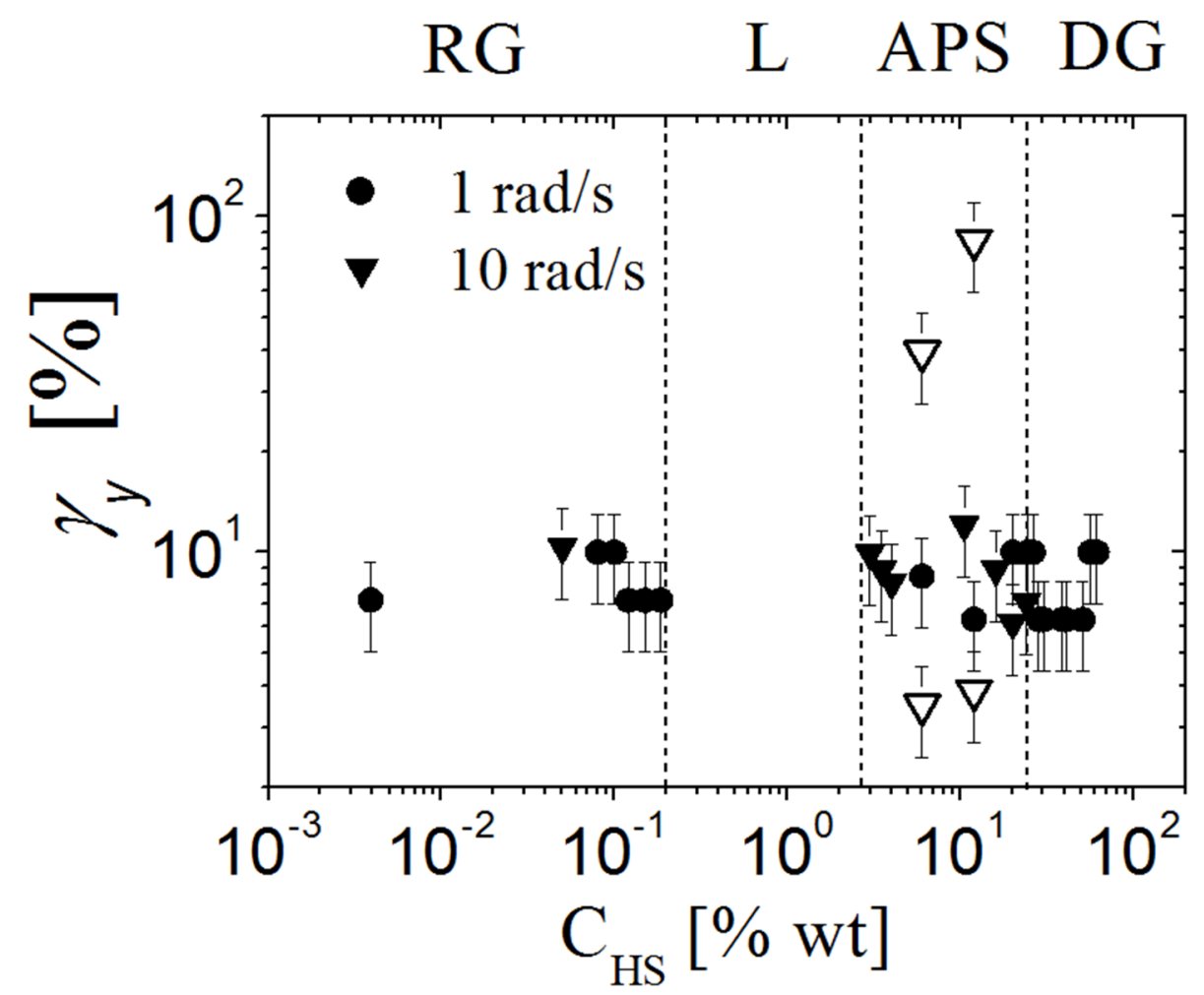

Figure 8: Yield strain in function of HS concentration for 2 different frequencies $(1 \mathrm{rad} / \mathrm{s}$ and $10 \mathrm{rad} / \mathrm{s})$. Open triangles refer to the yield strains measured for samples showing a "double-yield" behavior ( $\mathrm{c}_{\mathrm{HS}}=$ $6 \% \mathrm{wt}$ and $\mathrm{c}_{\mathrm{HS}}=12 \% \mathrm{wt}$ ).

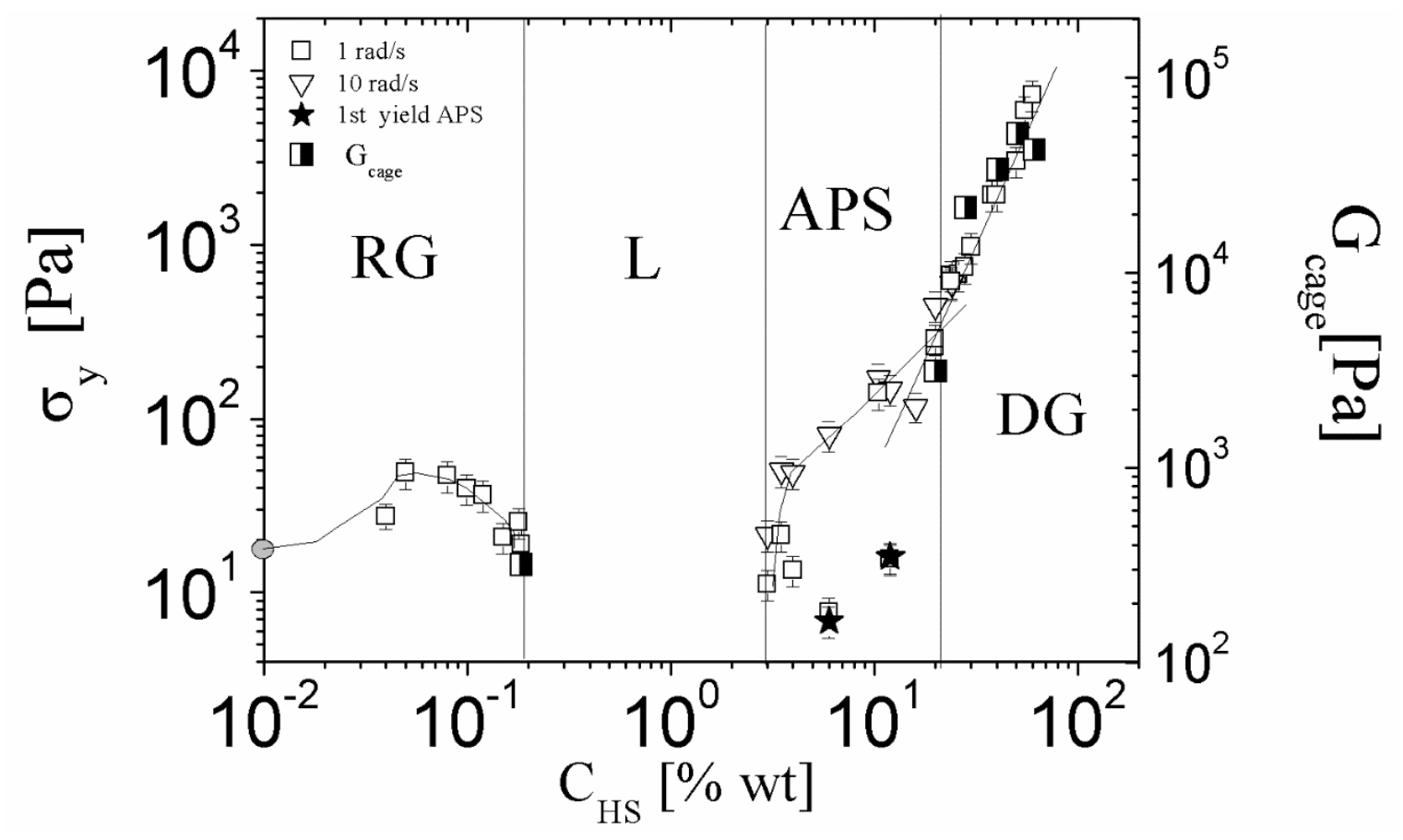

Figure 9: Yield stress (squares, triangles and stars) and cage modulus (half-filled squares) in function of HS concentration. Both yield stresses and cage modulus have been measured by performing dynamic strain sweeps. Lines in RP and APS regimes are drawn to guide the eye. Straight line in DG regime indicates power-law slope (see text). 
As already observed for the plateau modulus, the yield stress changes its $\mathrm{c}_{\mathrm{HS}}$-dependence for $\mathrm{c}_{\mathrm{HS}}>20 \%$, i.e., where we expect fully shrunk stars and the formation of a DG: it unambiguously increases more rapidly compared to the APS state as the content of HS is augmented. We find $\sigma_{\mathrm{y}} \sim \mathrm{cHS}{ }^{\beta}$ with $\beta=2.2 \pm 0.2$ in quantitative agreement with what we have found for Gp and $\gamma_{\mathrm{y}}$.

\section{III.3. Transient shear: start-up and residual stresses}

Figure 10 shows results from step-rate experiments for three different states, RG, APS and DG. Shear stress is normalized by the thermal modulus of a single star particle and the shear rate by its Brownian time $\tau_{B}=6 \pi R^{3} \eta /\left(k_{B} T\right)$. Here $\eta$ is the solvent viscosity, $k_{B}$ is the Boltzmann constant and $T$ is the absolute temperature. $R$ coincides to the hydrodynamic radius of the soft stars $R=R_{h}{ }^{\text {soft }}=45 \mathrm{~nm}$ for the sample in both RG $\left(c_{H S}=0.01 \% \mathrm{wt}\right)$ and APS $\left(c_{H S}=5 \% \mathrm{wt}\right)$ states, i.e. where no appreciable star shrinkage occurs [62]. The average radius $R=R_{C}\left(x_{H S}\left(\frac{1}{\delta^{3}}-1\right)+1\right)^{-1 / 3} \approx 11.6 \mathrm{~nm}$ [42], [62] for the sample in the DG state $\left(c_{H S}=50 \% \mathrm{wt}\right)$ has been used, where $R_{c}=16 \mathrm{~nm}$ is the radius of the collapsed soft stars, $R_{H S}=11.5$ $\mathrm{nm}$ is the hydrodynamic radius of HS-like stars, $x_{H S}=\frac{n_{s o f t} R_{C}^{3}}{n_{H S} R_{H S}^{3}+n_{s o f t} R_{c}^{3}}=0.95$ is the relative volume fraction of small HS-like stars and $\delta=0.71$ is the size ratio of the two type of stars in the DG state. The numbers of HS-like stars $n_{H S}$ and soft stars $n_{\text {soft }}$ in the sample are known from sample preparation.

After an initial elastic response $(\sigma \alpha \gamma)$, the mixtures undergo yielding unambiguously characterized by a stress overshoot and eventually a steady-stress is reached. Stress overshoot is present in both RG and DG and it is related to the deformation and distortion of the cages prior to yielding. Such effect is more pronounced for repulsive glassy states where more stress can be stored by the system before it fails, and is strongly dependent on the Péclet number $P e=\dot{\gamma} \tau_{B}$, where $\tau_{B}$ is the Brownian time of the single star.

The higher the Péclet number the higher the stress overshoot. In the double glass $\left(c_{H S}=50 \% \mathrm{wt}\right)$ the stress peak appears weaker because stars are completely shrunk and the system behaves like an asymmetric binary mixture of quasi-hard spheres, whose ability to store stress before yielding is sensibly reduced. 
The APS state does not exhibit any pronounced overshoot. Indeed, the stress evolution across the yield point is smoother and rather complex, as it has also been shown in the strain sweep measurements, where different length scales are involved, recalling the behavior of attractive colloidal systems.

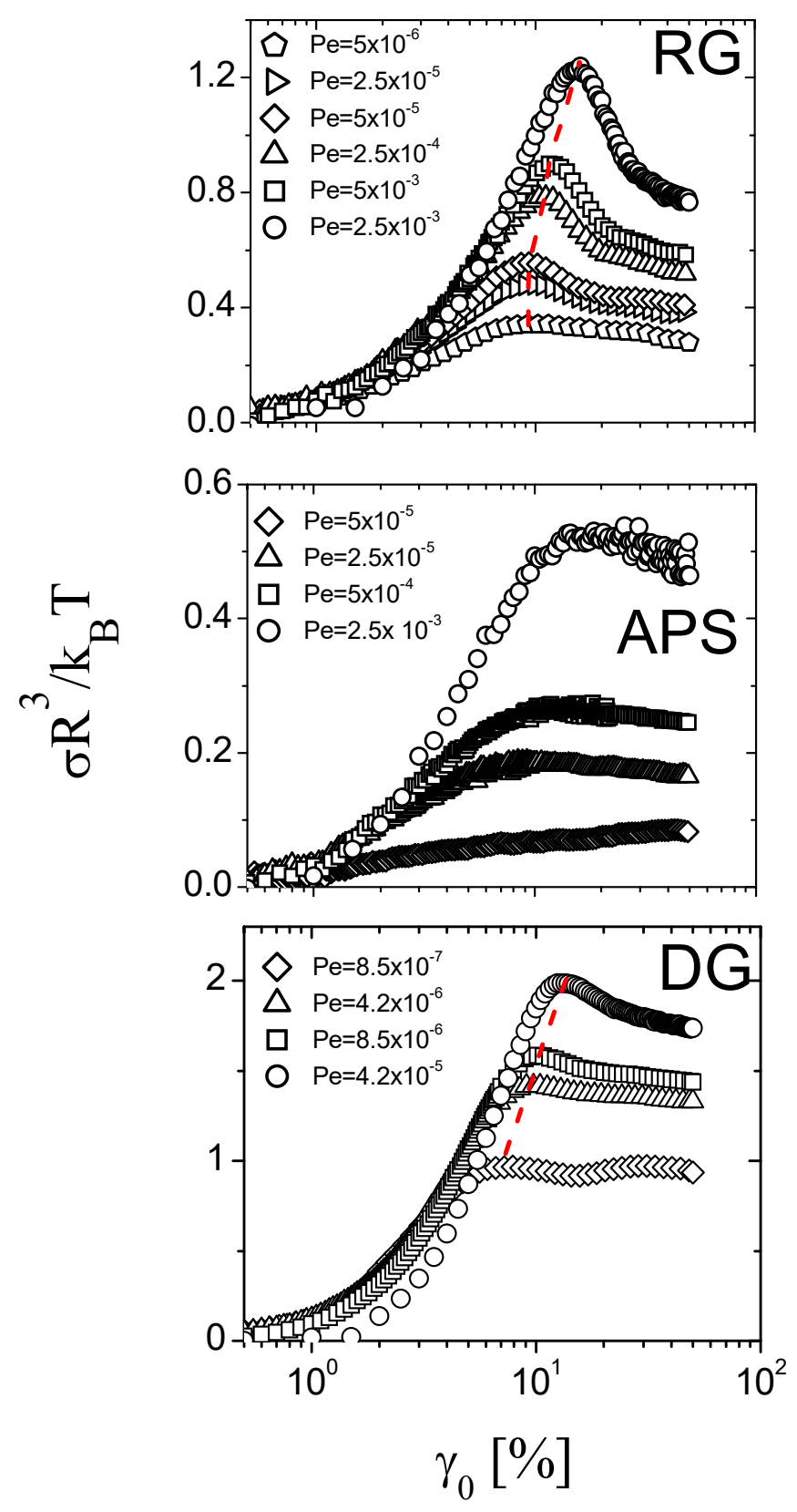

Figure 10: Step rate measurements for the three different solid states being, from the upper panel to the bottom one, respectively: RG ( $\mathrm{cHS}=0.01 \% \mathrm{wt})$, APS ( $\mathrm{cHS}=5 \% \mathrm{wt})$, DG ( $\mathrm{cHS}=50 \% \mathrm{wt}$ ). Shear stress is normalized by the thermal modulus of a single particle and the shear rate by the Brownian time (see text). Dashed lines in panels RG and DG indicate the evolution of peak stress with Pe. 
Figure $11(\mathrm{~A}, \mathrm{~B})$ shows the stress peaks normalized by the thermal modulus of a single particle $k_{B} T / R^{3}$ and the corresponding yield strain as function of the Péclet number.

We observe a weak dependence of the (normalized) yield stress peak with increasing Pe for both RG and DG $\left(\sigma_{y} R^{3} / k_{B} T \sim c H S^{0.2}\right)$. Interestingly, the normalized values of the peak stresses for RG and DG do not overlap, confirming that these states are indeed different in nature: yield stresses cannot be rescaled by applying simply the fluctuation-dissipation scaling. Furthermore, yield stresses and strains, obtained with oscillatory measurements at different frequencies (DSS tests) have been added to the latter plots displaying a good agreement with transient measurements. Strain values encompass a region around $10 \%$ strain with an increasing trend with Pe (and a slope of about 0.15 , i.e., similar to that of stress), in agreement with other soft colloidal systems [66], [42], [49], [78].

When the normalized steady-state stress is plotted versus Pe (Figure 12A) further differences appear among the three different states: quite interestingly, APS displays the weakest plateau stress and the strongest dependence on Pe. On the other hand, the selected DG state shows high normalized stresses, well above unity and above the values measured for the initial RG state. In an analogous fashion, the steady shear and dynamic viscosities can be plotted against Pe (Figure 12B). In fact, when oscillatory complex viscosity data are plotted versus $P e_{\omega}=6 \pi \eta \omega \gamma_{0} R^{3} / k_{B} T$ the empirical Delaware-Rutgers [79] rule is validated: the steady state shear viscosity corresponds to the complex viscosity modulus when plotted versus a properly scaled ratio between the microscopic diffusion and the advection time. Such correspondence is not necessarily fulfilled [80] for all structurally complex polymer-based system and our result must be considered as specific of the present soft colloidal mixtures. Despite of that the validity of the Delaware-Rutgers rule suggests that our mixtures are not characterized by large structural heterogeneities while being amorphous and characterized by very weak ageing. 


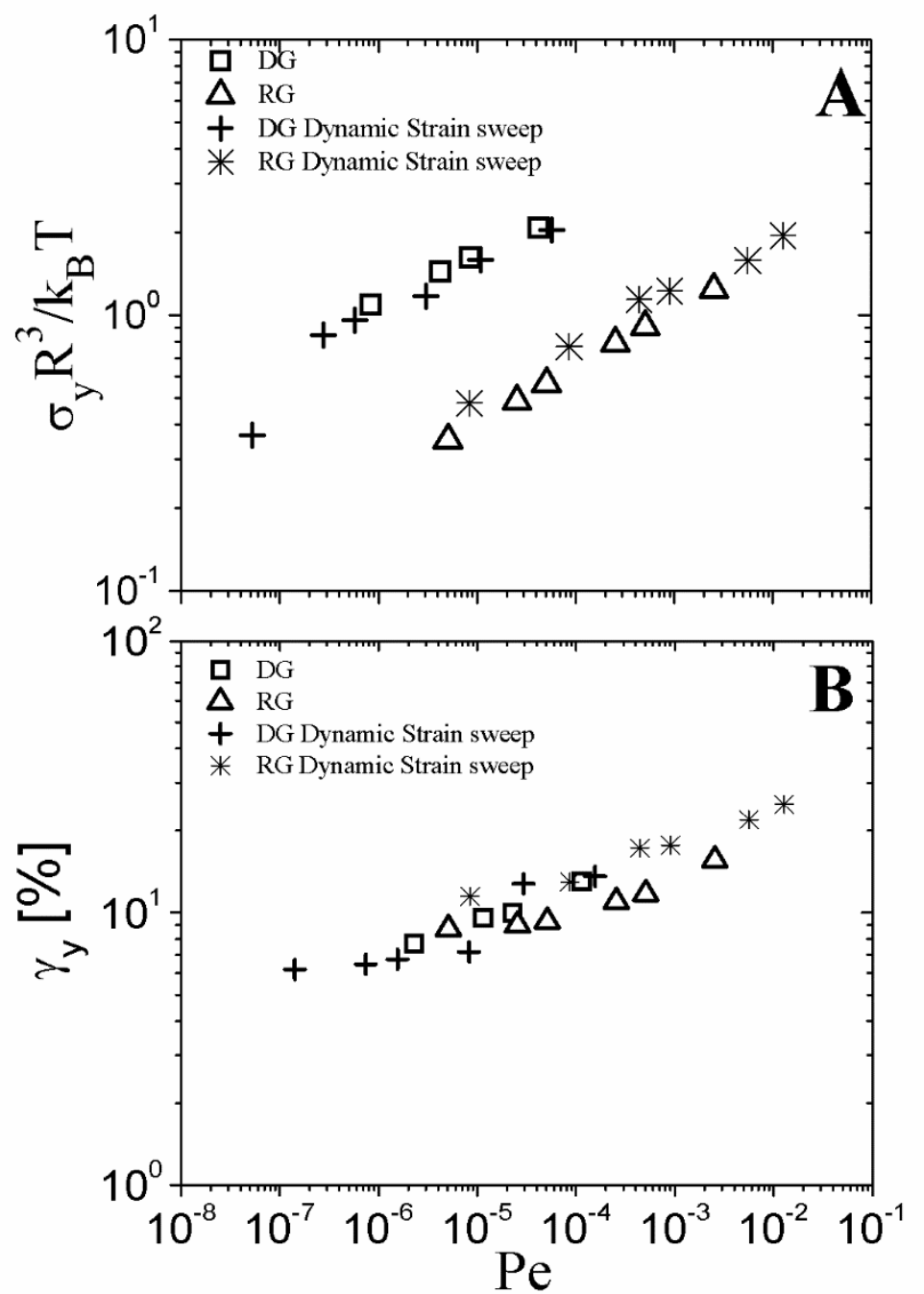

Figure 11: (A) Normalized stress peak and (B) strain peak for RG-state ( $c_{H S}=0.01 \% \mathrm{wt}$ ) and DG-state $\left(c_{H S}=50 \% \mathrm{wt}\right)$ as function of Pe. Oscillatory yield stresses and corresponding yield strains are also shown. 

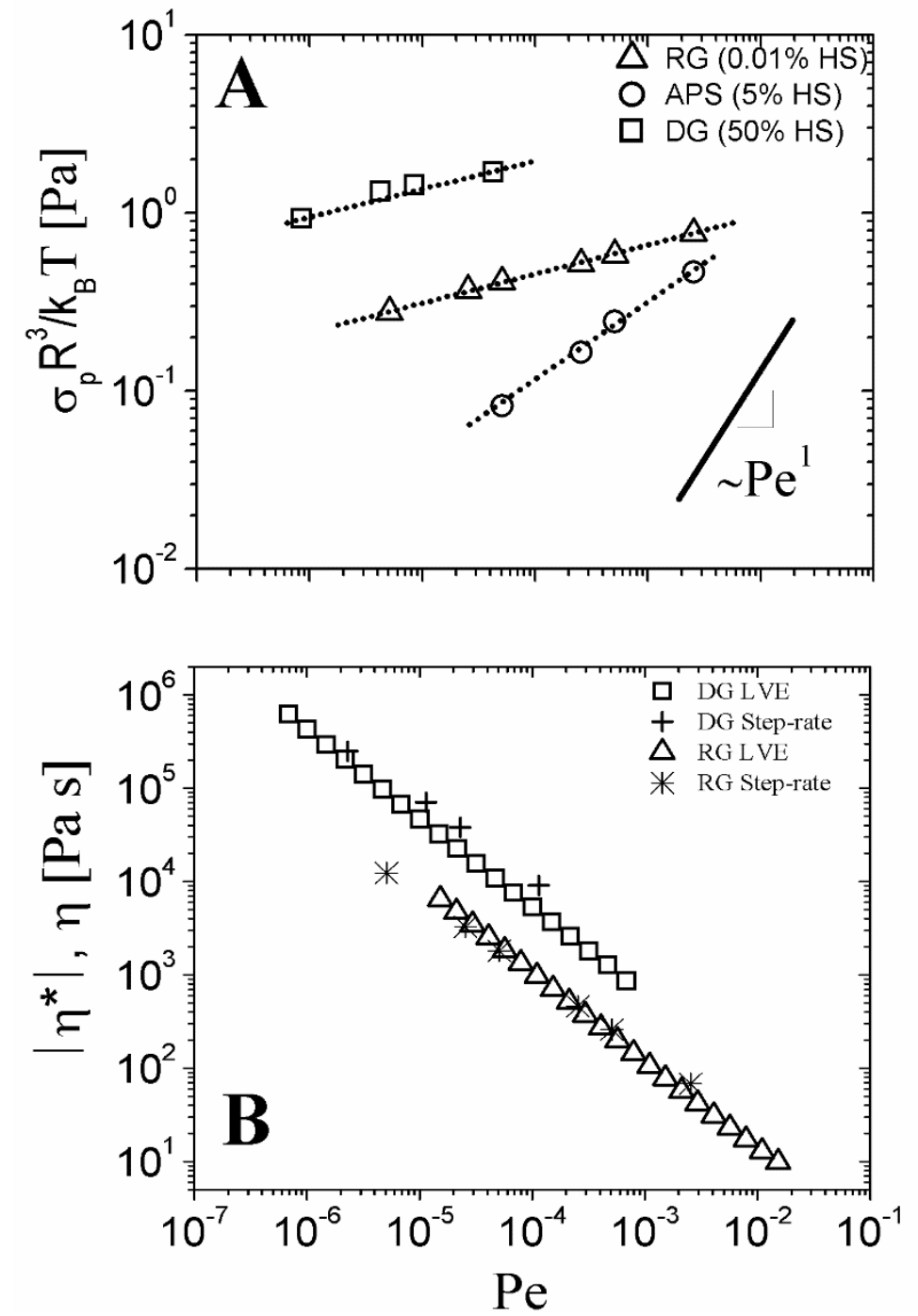

Figure 12: A) Normalized steady-state stress for RG (0.01\% HS), APS (5\% HS) and DG (50\% HS). B) Absolute value of the complex viscosity and steady-state viscosity for RG $(0.01 \% \mathrm{HS})$ and DG $(50 \%$ HS). as function of Pe. Dotted black lines, in panel A, are drawn to guide the eye.

Next, we followed the relaxation processes after flow cessation in the three different states, RG, APS and DG. Indicative results of stress relaxation tests for the different states and concentrations of HS-like stars are shown in Figure 13. The stress values are normalized by $\sigma_{\mathrm{p}}$, the stress corresponding to the steady-state stress (plateau) measured at the long time limit of $\sigma(t)$ in transient tests performed at different shear rates $\dot{\gamma}$. The probed $\operatorname{RG}\left(c_{H S}=0.01 \%\right)$ and APS state $\left(c_{H S}=5 \%\right)$ relax after $100 \mathrm{~s}$ : the accumulated stress due to translational and rotational strain-induced motion relaxes after 100 sun like hard sphere glasses or jammed microgel suspensions which are characterized by non-negligible residual stresses [81], [82], [83]. On the other hand, the DG state is characterized by stresses not fully relaxed 
after $100 \mathrm{~s}$. Such finding suggests that particle softness is important in determining the full relaxation of accumulated stress in colloidal glasses and also indirectly confirms that DG state is dominated by the frustrated dynamics of hard particles (HS-like stars and collapsed soft stars).Moreover, it is worth mentioning that for high shear rates, DG samples were found to be mechanically instable: for $c_{H S}>20 \%$ wt edge fracture systematically occurred for shear rates higher than $0.5 \mathrm{~s}^{-1}$ (data not shown), limiting the range of shear rates that were experimentally accessible (see inset Figure 13C).

To better compare the behavior of the different states, we report in Figure 14 the normalized stress $\sigma / \sigma_{p}$ plotted in function of their scaled time $(t \dot{\gamma})$ [81]. As already observed for other colloidal glasses and in simulations [81], [84], [85], the stress relaxation curves collapse into master curves that help distinguishing the features of each state: (i) RG and APS states relax to zero stress after t 100s; (ii) On the other hand, for the probed sample in DG state, stresses do not fully relax, signaling the presence of residual stresses. This representation allows us to assign to the shear-rate the role of scaling parameter in the time domain for stress relaxations in soft glasses, in accordance with recent work on glassy hard spheres [81]. This finding suggests that hard repulsions are very important to generate stress frustration in repulsive colloids; such is the case of the DG state, where soft stars shrink due to the presence of hard-sphere-like stars, their size ratio approaches a value of 0.71 and all types of particles interact via hard repulsions [62]. 


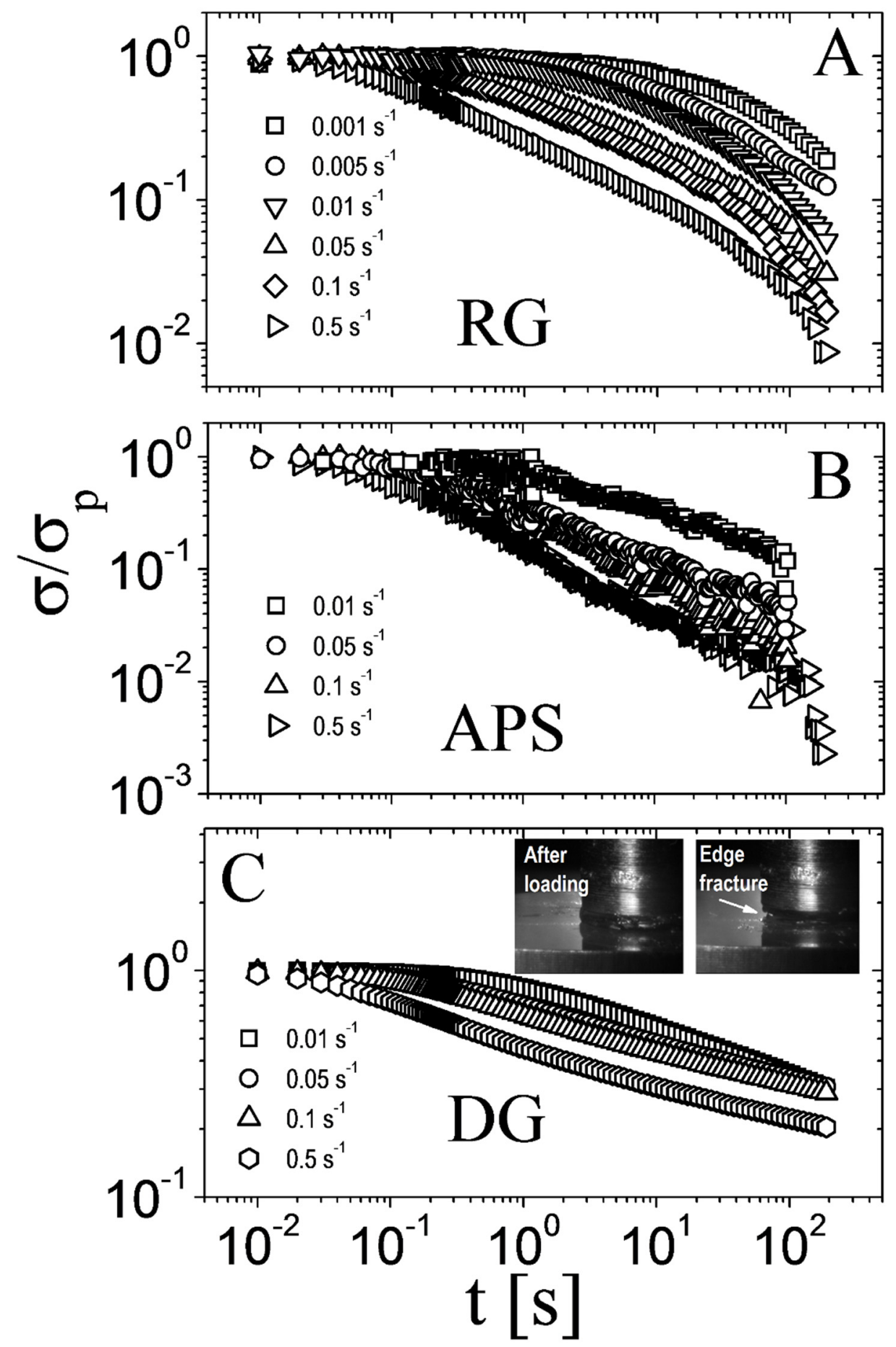

Figure 13: Stress relaxation following flow cessation at different shear rates for the three different solid states discussed in the main text.Panel A: $c_{H S}=0.01 \%$ wt. Panel B: $c_{H S}=5 \%$ wt. Panel C: $c_{H S}=50 \%$ wt. The inset in panel C shows the onset of edge fracture observed in DG samples for $\dot{\gamma}>0.5 \mathrm{~s}^{-1}$ (see text). 


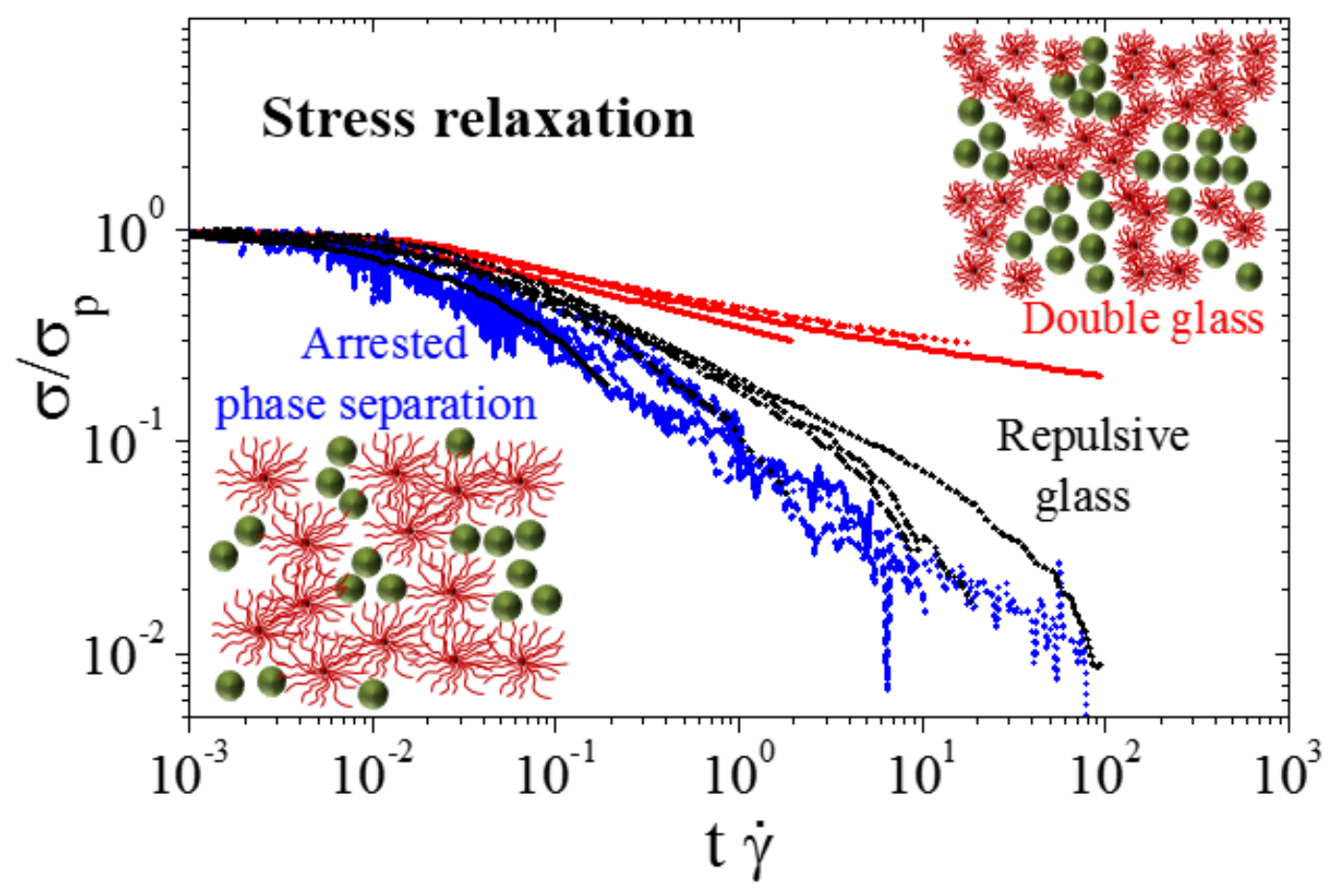

Figure 14: Normalized stress relaxation for RG-state $\left(\mathrm{c}_{\mathrm{HS}}=0.01 \% \mathrm{wt}\right)$, APS-state $\left(\mathrm{c}_{\mathrm{HS}}=5 \% \mathrm{wt}\right)$ and DG-state $\left(c_{H S}=50 \% \mathrm{wt}\right)$ as function of the normalized time $\dot{\gamma} t$ (where the shear rate refers to the imposed flow before cessation).Different lines refer to the different shear rates at the time of cessation. The cartoons illustrate schematically the APS and DG states.

\section{III.4. LAOS}

Figure 15depicts LAOS results for the RG, APS and DG states at the same Pe and $\gamma>\gamma_{\mathrm{y}}$. Panels $\mathrm{A}_{1}, \mathrm{~A}_{2}, \mathrm{~A}_{3}$ and $\mathrm{B}_{1}, \mathrm{~B}_{2}, \mathrm{~B}_{3}$ report the Lissajous-Bowditch curves in two distinct representations: panels A show the stress plotted vs the strain (elastic representation), while in panels B the stress is plotted vs the strain rate (viscous representation). Panels $C_{1}, C_{2}$ and $C_{3}$ show selected waveforms $\sigma(t)$ for the three states, which exhibit different responses when large-amplitude deformations $\left(\gamma_{0}=100 \%\right)$ are applied at $\omega=1 \mathrm{rad} / \mathrm{s}$. In the case of $\mathrm{RG}$, a weak albeit detectable stress overshoot appears in the elastic response (see arrows in Figure 15, A1). Concomitantly, a secondary loop in the viscous response (see arrows in Figure 15) [86] appears. The stress peak is also visible in the waveform, as indicated by the arrows in panel $\mathrm{C}_{1}$. Such behavior has been already reported for interdigitating hairy particle suspensions in the glassy state, like star polymer solutions [87]. On the other hand, APS and DG do not exhibit any stress overshoot and consequently any secondary loop, consistent with results obtained for glassy microgel 
suspensions without dangling arms or glassy hard spheres for $\gamma<10 \gamma_{\mathrm{y}}[72]$, [88]. Hence, we tentatively associate this behavior with the absence or significantly reduced arm interpenetration with increasing HS concentration. This can be also clearly evidenced if the elastic and viscous components of the stress are decoupled following the analysis of Cho et al. [89], [90], who showed that an an strain amplitude the stress can be expressed as a sum of two functions: one exhibiting odd symmetry with respect to the strain and even symmetry with respect to strain rate (i.e., the elastic component of the stress, $\sigma^{\prime}$ ), and another one exhibiting even symmetry with respect to the strain and odd symmetry with respect to strain rate (i.e. the viscous component of the stress, $\sigma^{\prime \prime}$ ). In Figure 16 we plot the elastic component of the stress normalized by its maximum value $\sigma^{\prime} / \sigma_{\max }^{\prime}$ for different hard-star concentrations spanning all the solid-like states of the mixtures (RG, APS, DG). An unambiguous stress peak characterizes the samples in the RG state. For selected samples we have also determined the cage modulus from the slope of the stress-strain curve at zero stress[87], [24]: $G_{c a g e}=d \sigma /\left.d \gamma\right|_{\sigma=0}$, a quantity nearly independent from the strain amplitude above the yield point for all the samples investigated. The result is reported in Figure 9 together with the yield stress data. As expected, $G_{c a g e}$ reflects both the dependences of $\sigma_{y}$ and $G_{p}$ on the HS-like star content.

The effect of the Péclet number has been investigated by changing the frequency of the LAOS tests at fixed strain amplitude. The result is reported in Figure 17: a distortion of the typical parallelogram pattern indicative of an intra-cycle sequence of elastic-plastic response at $\gamma_{0}>\gamma_{y}$ is observed at high frequency for the RG state indicative of highly distorted cages. Indeed, Brownian Dynamics (BD) simulations with hard sphere glasses attribute the distortion of the stress in the quadrants II and IV in the elastic representation of LAOS to the structural anisotropy of cages at the point of strain reversal that reduces the number of contacts between neighboring particles as the strain increases again [88], [91]. Such distortion does not appear in the APS state, apparently due to the persistence of isotropic structures under large deformation and Péclet numbers. Since edge fracture has been observed for samples in the DG state, the relevant investigation was limited to frequencies up to 10 
$\mathrm{rad} / \mathrm{s}$, without significant distortion of the patterns being observed. Stress data are normalized by the maximum stress detected during LAOS experiments, as reported in Figure 15.

In addition to the above analysis, we have characterized the nonlinear response of the mixtures by FT-rheology, where the time dependence of the stress response was decomposed Fourier modes.

In all cases under investigation the signal-to-noise ratio $(\mathrm{S} / \mathrm{N})$ was higher than $\sim 10^{5}$ and increased by passing from repulsive (soft) glasses to double (hard) glasses up to $\sim 10^{7}$.

Figure 18 depicts a representative Fourier spectrum for a sample in the RG state $\left(c_{H S}=0 \%\right)$ and one in the DG state $(c H S=50 \%)$. As expected, in both cases the contribution of even harmonics is negligible and the stress response is a periodic odd function of strain. This has been observed for all HSlike stars concentrations investigated. In what follows we take a closer look at the amplitude of first two higher harmonics $I_{3}$ and $I_{5}$ and their strain amplitude dependence. As a matter of fact, $I_{3} / I_{1}$ and $I_{5} / I_{1}$ have been used often in order to quantify the nonlinear behavior in LAOS flow[92],[93], [94]. For polymeric or wormlike micellar systems, results from LAOS experiments over a wide range of applied deformation amplitudes revealed respective scaling laws $I_{3} / I_{1} \propto \gamma_{0}^{2}$ and $I_{5} / I_{1} \propto \gamma_{0}^{4}$ [95], [96], [93]. They were supported by simulations using various nonlinear constitutive equations including the Giesekus [97], pom-pom [98], and molecular stress function (MSF) models [99]. Indeed, the stress response of a generic viscoelastic material can be expressed by defining a set of nonlinear viscoelastic moduli $G_{m n}^{\prime}(\omega), G_{m n}^{\prime \prime}(\omega)$ and reads [100] as:

$$
\sigma(t)=\sum_{m=1, \text { odd } n=1, \text { odd }}^{+\infty} \sum_{0}^{m}\left[G_{m n}^{\prime}(\omega) \sin (n \omega t)+G_{m n}^{\prime \prime}(\omega) \cos (n \omega t)\right]
$$

This expression nicely separates the strain dependence from the frequency dependence and yields the observed scaling $I_{3} / I_{1} \propto \gamma_{0}^{2}$ and $I_{5} / I_{1} \propto \gamma_{0} 4$ in the limit of small strain amplitudes [95].

Motivated by these scaling laws, in an effort to obtain universal measures of nonlinear response, Hyun and Wilhelm [95] and Ahirwal et al. [93] introduced two new nonlinear parameters $Q_{3}=\frac{I_{3}}{I_{1} \gamma_{0}{ }^{2}}$ 
and $Q_{5}=\frac{I_{5}}{I_{1} \gamma_{0}{ }^{4}}$,where $\gamma_{0}$ is expressed in absolute units of strain. The $Q$-parameters in the limit of low strain amplitudes approached constant values, which were called "intrinsic nonlinearity parameters", $Q_{3}^{0}=\lim _{\gamma_{0} \rightarrow 0} Q_{3}$ and $Q_{5}^{0}=\lim _{\gamma_{0} \rightarrow 0} Q_{5}$. These parameters were subsequently used in order to characterize the effect of branching in linear and comb polystyrenes [95], the effect of polydispersity in polyethylene melts [101], the nonlinear viscoelasticity of blends of linear and branched polyethylene and polypropylene [93] and the effect of chain length and polydispersity on the nonlinear viscoelasticity of linear polymer melts of different chemistry [102]. However, despite their relevance in the phenomenological characterization of the nonlinear viscoelasticity of materials, such sensitive nonlinear response parameters have not been extracted for colloidal systems.

Figure 19-Adepicts the normalized third, fifth and seventh harmonic in the RG regime, over a wide range of hard-star concentrations $c H S$. The data suggest that higher harmonics are not affected by the weakening of the RG due to the addition of hard particles. The above mentioned phenomenological scalings $I_{3} / I_{1} \propto \gamma_{0}^{2}$ and $I_{5} / I_{1} \propto \gamma_{0}^{4}$ characterize the strain dependence of the first secondary two harmonics at low strains $\left(\gamma<\gamma_{y} \approx 10 \%\right)$. On the other hand, much weaker dependencies have been observed above the yield point $\left(\gamma>\gamma_{y}\right)$, suggesting that upon yielding the nonlinear response is only weakly affected by the amplitude of the deformation.

We emphasize the above point by plotting the two nonlinear parameter $\mathrm{s} Q_{3}$ and $Q_{5}$ (Figure 19-B) for all available data in the three states RG, APS and DG: as expected, a plateau characterizes their strain dependence at low strains, from which we extracted the two intrinsic nonlinearity parameters by averaging the data within the interval $5 \% \leq \gamma_{0} \leq 17 \%: Q_{3}^{0}=6 \pm 3$ and $Q_{5}^{0}=81 \pm 65$. The latter can be expressed as $Q_{3}^{0}(\omega)=\frac{\left|G_{33}^{*}(\omega)\right|}{\left|G_{11}^{*}(\omega)\right|}$ and $Q_{5}^{0}(\omega)=\frac{\left|G_{55}^{*}(\omega)\right|}{\left|G_{11}^{*}(\omega)\right|}$, where $\left|G_{11}^{*}(\omega)\right|,\left|G_{33^{*}}(\omega)\right|$ and $\left|G_{55}{ }^{*}(\omega)\right|$ are the 
linear complex modulus, the third nonlinear complex modulus and the fifth nonlinear complex modulus, respectively, and represent the coefficients of the power series of the shear stress in equation (1), as introduced by Pearson and Rochefort [103].

We remark that the values obtained here are much higher than those previously obtained in blends of linear and branched chains [93], [102], linear and comb polymer melts [95], for which typical values of the intrinsic nonlinearity parameters range from $10^{-3}$ to $10^{-2}$. Moreover, both $Q_{3}{ }^{0}$ and $Q_{5}{ }^{0}$ are larger than 1 , suggesting that at $\omega=1 \mathrm{rad} / \mathrm{s}$ the third and the fifth nonlinear complex moduli, $\left|G_{33}{ }^{*}(\omega)\right|$ and $\left|G_{55}{ }^{*}(\omega)\right|$ respectively, prevail over the first complex modulus for all mixtures investigated: we get $\left|G_{55}{ }^{*}(\omega)\right|>\left|G_{33}{ }^{*}(\omega)\right|>\left|G_{11}{ }^{*}(\omega)\right|$, a result that has not been observed previously in other complex polymerbased fluids and that can be interpreted as the tendency of soft colloid-based solids to oppose more resistance to high harmonics than the first one. This suggests that excluded volume effects and density heterogeneities due to particle topology are radically different, as expected, from those characterizing polymer melts. As a matter of fact, in the nonlinear regime the component of the stress given by the $3 \mathrm{rd}$ and 5th harmonics can be written (to the leading order in $\gamma_{0}$ ) as $\sigma_{3}=G_{33}^{*} \gamma_{0}{ }^{3}$ and $\sigma_{5}=G_{55}^{*} \gamma_{0}^{5}$, indicating that the intrinsic resistance of the present colloidal mixtures to an imposed oscillatory shear strain containing high-order harmonics increases as the wave number of the imposed strain increases. Our analysis poses new questions, for example whether such difference between polymer melts, for which $\left|G_{55}{ }^{*}(\omega)\right|<\left|G_{33}{ }^{*}(\omega)\right|<\left|G_{11}{ }^{*}(\omega)\right|[102]$, [93], [95], [101] and colloidal suspensions is generic (i.e., whether it is qualitatively independent from the type of colloids or simply specific to the present mixtures). In this respect, it is worth noting that similar scaling and intrinsic nonlinearity values can be obtained by calculating $Q_{3}^{0}$ and $Q_{5}^{0}$ starting from the amplitude of high-order harmonics reported by Brader et al.[104] for dense colloidal suspensions of core-shell latex particles and that this finding is further corroborated by Mode Coupling Theory (MCT) [104]. This appears to suggest a generic behavior of the intrinsic nonlinearity parameters, the latter being able to discern clearly the topology and the very general features of the constituents of different viscoelastic solids. Nevertheless, despite the 
strong evidence from the present observations, further work is needed to elucidate such point, which however is beyond the scope of this paper.
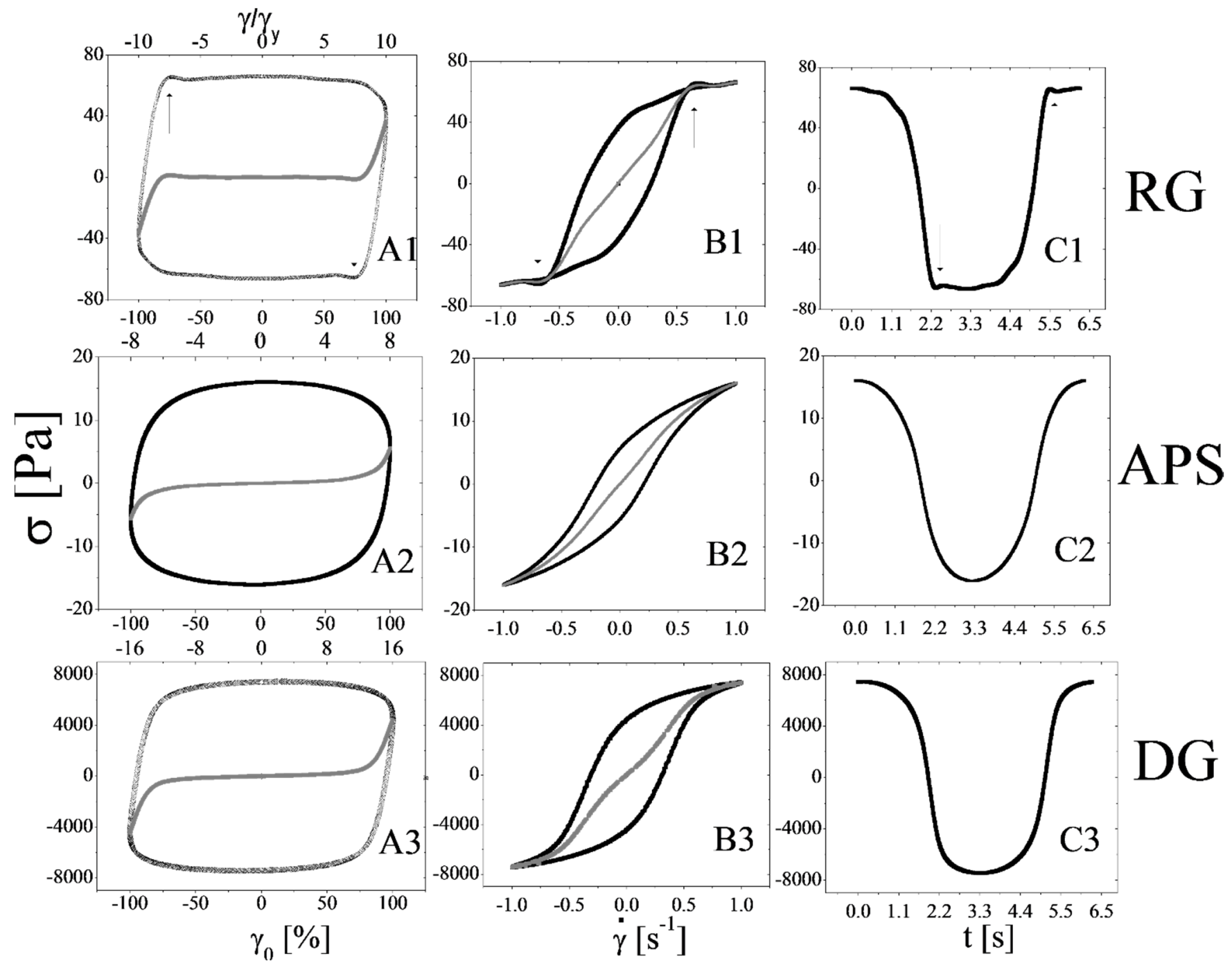

Figure 15: Elastic representation (Panels A) and viscous representation (Panels B) of LAOS experiments $\left(\gamma_{0}=100 \%\right.$ and $\left.\omega=1 \mathrm{rad} / \mathrm{s}\right)$ for selected samples in RG-state $\left(c_{H S}=0.08 \%\right.$ wt - Panels A1, B1), APS-state $\left(c_{H S}=10 \% \mathrm{wt}\right.$ - Panels A2, B2) and DG-state $\left(c_{H S}=50 \%\right.$ wt- Panels A3, B3). Panels C1, $\mathrm{C} 2$ and $\mathrm{C} 3$ show the correspondent stress-waveforms. Grey lines show the elastic (Panels A1, A2, A3) and the viscous (Panels B1, B2, B3) components of the stress. 


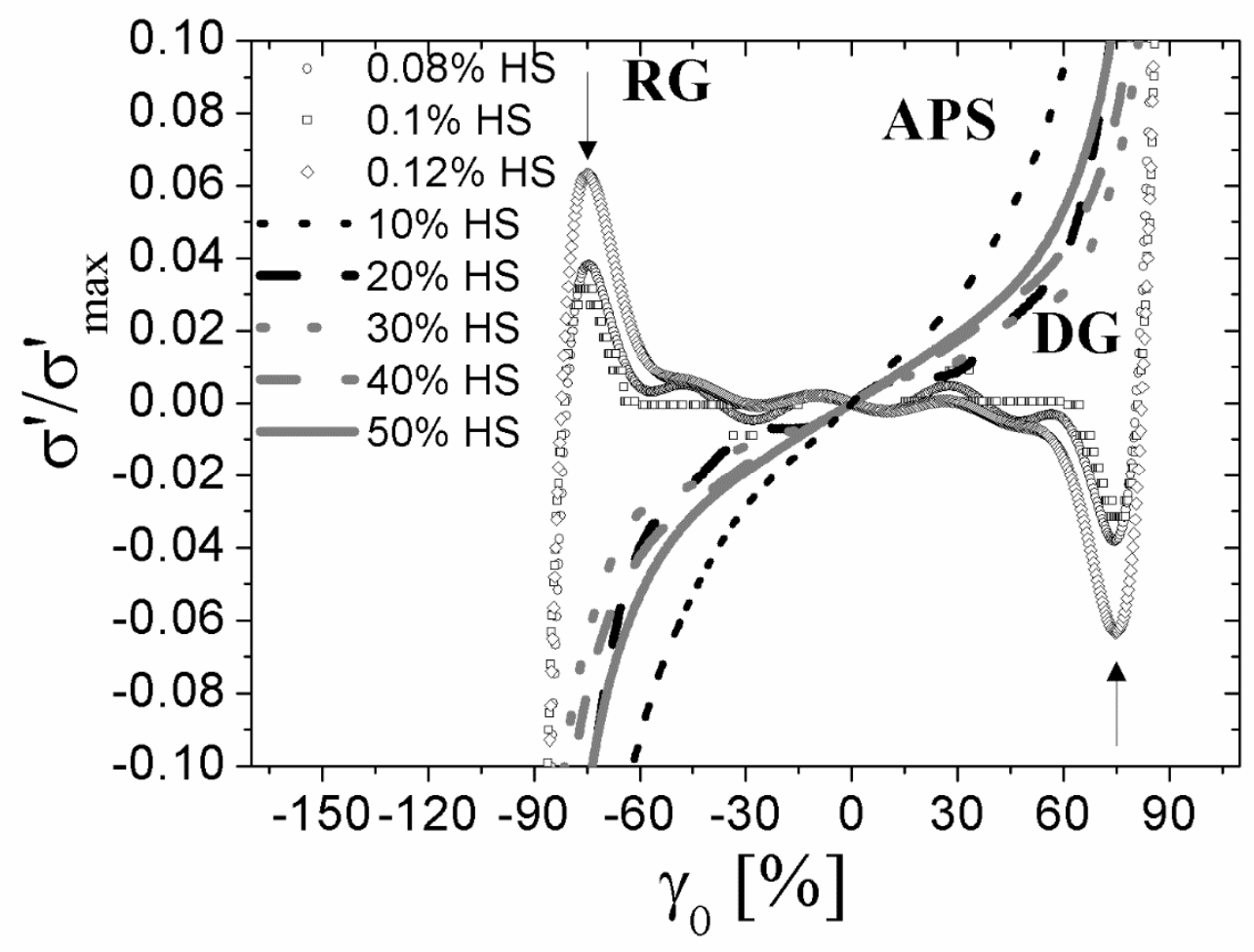

Figure 16: Elastic component of the stress normalized by its maximum value $\sigma^{\prime} / \sigma^{\prime} \max a t \omega=1 \mathrm{rad} / \mathrm{s}$ and $\gamma_{0}=100 \%$ (corresponding to $\mathrm{Pe}=0.005$ for $\mathrm{RG}$ and APS and $\mathrm{Pe}=8.5 \times 10^{-5}$ for DG) for different HS-like star concentration as indicated in the figure. 


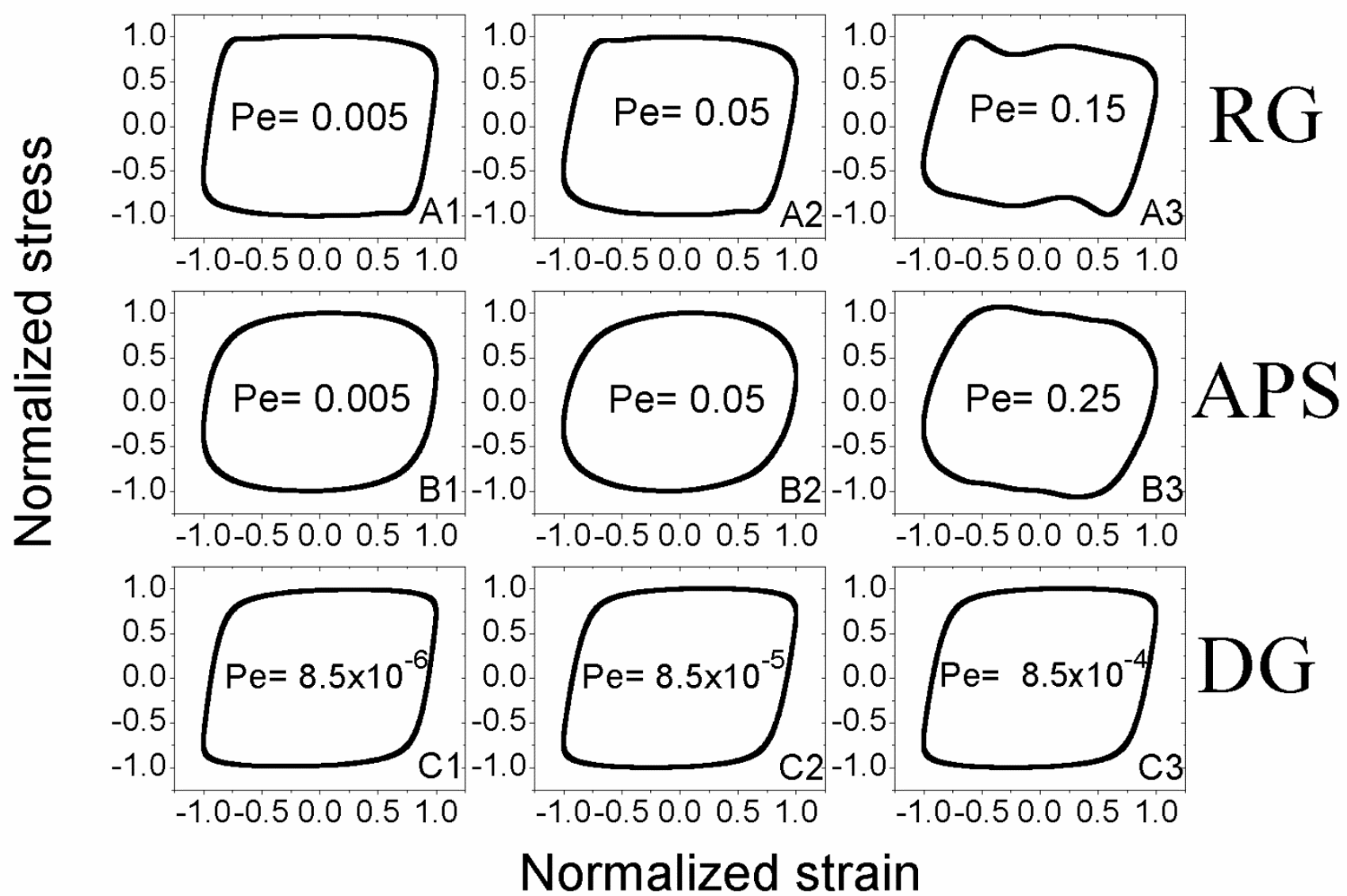

Figure 17: Elastic representation of Large Amplitude Oscillatory (LAOS) experiments at $\gamma_{0}=100 \%$ and different Pe (frequencies) for selected samples in RG-state ( $c_{H S}=0 \% \mathrm{wt}$ - A panels), APS-state $\left(c_{H S}=10\right.$ $\% \mathrm{wt}-\mathrm{B}$ panels) and DG-state ( $c_{H S}=50 \% \mathrm{wt}-\mathrm{C}$ panels). Stress data are normalized by the maximum stress detected during LAOS experiments, as reported in Figure 15. 


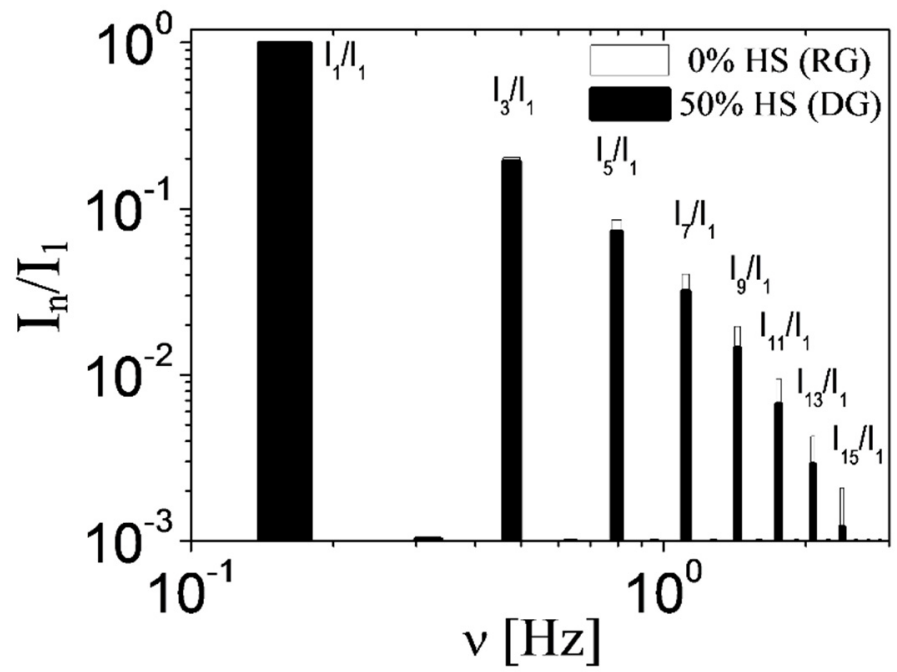

Figure 18: Fourier spectrum of the oscillatory shear response of a sample in the RG state ( $\left.c_{H s}=0 \%\right)$ and DG state $\left(c_{H S}=50 \%\right)$ at $\gamma_{0}=100 \%$ and $\omega=1 \mathrm{rad} / \mathrm{s}$ (corresponding to $\mathrm{Pe}=0.005$ for $\mathrm{RG}$ and $\mathrm{Pe}=8.5 \times 10^{-5}$ for DG).
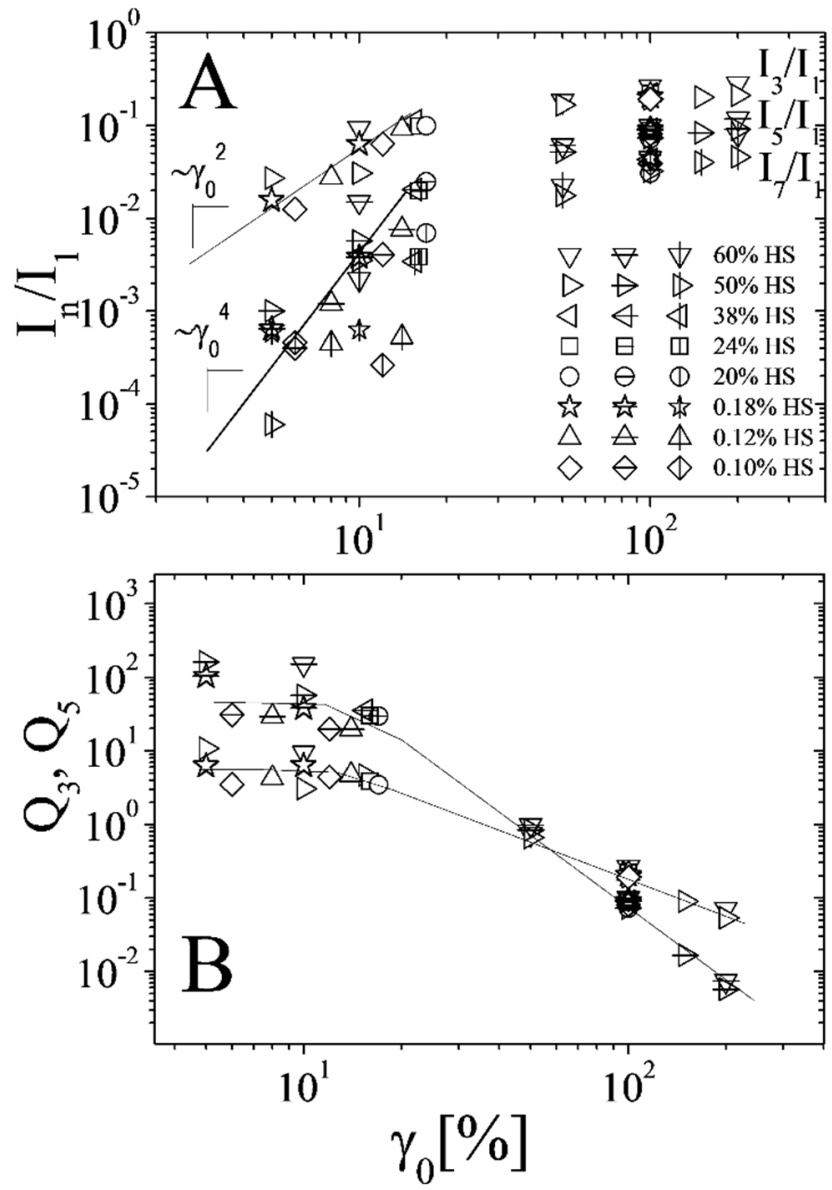

Figure 19: Strain dependence of the normalized $3^{\text {rd }}$ (open symbols), $5^{\text {th }}$ (open symbols horiz. cut) and $7^{\text {th }}$ (open symbols vert. cut) harmonic for different HS concentrations at $\omega=1 \mathrm{rad} / \mathrm{s}$ (Panel A). Different symbols correspond to different HS concentrations as indicated in the figure. The expected scaling for low strains is also shown for the $3^{\text {rd }}$ and $5^{\text {th }}$ harmonic. Panel B shows the nonlinear parameters $Q_{3}\left(\gamma_{0}\right)$ 
and $\mathrm{Q}_{5}\left(\gamma_{0}\right)$ (same symbols as Panel A). Solid lines are only a guide for the eye. The data span the three solid-like states encountered (RG, APS, DG).

\section{Conclusions and emerging picture}

We have presented a detailed comparative rheological investigation of binary colloidal mixtures involving a large soft (initially glass) and a small hard (additive) component. To this end, we use two different well-characterized star polymers, a soft (lower functionality, larger arm molar mass) and a hard (higher functionality, smaller arm molar mass). In this case, due to the osmotic forces induced by the hard star and leading to soft star compression, the size ratio varies from about 0.25 to about 0.71 , as the hard star fraction increases at constant soft star volume fraction. As a consequence of the coupling of softness and osmotic forces, different kinetic states are found and discussed, including repulsive single glass (RG), liquid, arrested phase separation (APS) and double glass (DG). These states exhibit different linear viscoelastic response. A systematic set of nonlinear rheological data has been obtained, under transient shear and dynamic oscillatory conditions. In particular, we have investigated the start-up of stress at constant rate, its relaxation upon flow cessation, and the response under large amplitude oscillatory shear. Distinct features are identified and serve as signatures of the different states. DG exhibits a larger yield stress compared to RG and APS. It is also characterized by a pronounced residual stress, unlike the other states. Yield strains are the same for all states. Two-step yielding was evidenced for APS for the investigated frequencies. The origin of the differences appears to lie in the more compact conformation of the osmotically shrunk soft stars in DG, rendering that state akin to a hard colloidal glass. This is in contrast to the other states where arm interdigitation plays a role. For both soft star- and hard star-dominated mixtures (i.e., RG and DG, respectively), the high values of the intrinsic nonlinearity parameters $\left(Q_{3}, Q_{5}>1\right)$ are in agreement with those recently found for hard sphere and core-shell particle glasses, and in stark contrast with the very low intrinsic nonlinearity characterizing linear and branched polymer melts. The latter are characterized by broader linear regime $\left(\gamma_{\mathrm{y}} \sim 100 \%\right)$ when compared to colloidal suspensions $\left(\gamma_{\mathrm{y}} \sim 10 \%\right)$ and by a consequent low values of the 
intrisic nonlinearity parameters. In other words $Q_{3}^{0}$ and $Q_{5}^{0}$ tell how much a system can be deformed before it shows non-negligible higher harmonics: systems with low nonlinearity parameters will remain in the linear regime for a wide range of strain amplitudes and vice-versa. This reveals the "colloidal nature" of our mixtures at any composition. Finally, large amplitude oscillatory tests point out the keyrole played by mutual interdigitation on the global stress response of the mixtures and the occurrence of intra-cycle stress overshoots.

Our results provide strong evidence of the power of entropic mixing as a means to tailor the properties of soft composites and unambiguously show that the compositional tuning of mixtures of soft and hard particles represents a unique pathway to systematically explore the rheological transition from ultra-soft to hard amorphous solids. A special note on the DG state is in order: Inspired by remarkable recent work with microgels [75] where entropic glass and jammed glass were identified and distinguished in terms of their rheology, it is tempting to associate DG with conditions of jammed state, but this requires more work and represents a future direction. In conclusion, we hope that the present work offers insights into the better understanding of hard-soft colloidal mixtures where osmotic particle compression and depletion-mediated phase separation should be considered. In addition, given that the state behavior of such a system is accurately predicted by means of MCT and simulations [61], [62], predicting the unambiguous rheological features identified in this work with the same tools is a formidable challenge. Finally, the present results are important ingredients for molecular design of soft composites with desired and tunable flow properties.

\section{ACKNOWLEDGMENTS}

We thank Abdul Munam, John Marakis and Antje Larsen for assistance with initial sample synthesis, rheological and DLS measurements, respectively. Financial support from the EU (FP7 Infrastructure ESMI GA262348, ITN SOMATAI GA316866 and Horizon 2020 COLLDENSE GA642774) and the Natural Science and Engineering Research Council of Canada (NSERC) is gratefully acknowledged. 


\section{REFERENCES}

[1]. Quirk, R. P. K., J., "Recent Advances in Thermoplastic Elastomer Synthesis," Rubber Chemistry and Technology 64, 450-468 (1991).

[2]. Hadjichristidis, N. P., M.; Pispas, S.; Iatrou, H. , "Polymers with complex architecture by living anionic polymerization," Chemical Reviews 101, 3747-3792 (2001).

[3]. Wu, W. W., W.; Li, J., "Star polymers: Advances in biomedical applications," Progress in Polymer Science 46, 55-85 (2015).

[4]. Ren, J. M., T. G. McKenzie, Q. Fu, E. H. H. Wong, J. Xu, Z. An, S. Shanmugam, T. P. Davis, C. Boyer and G. G. Qiao, "Star Polymers," Chemical Reviews 116, 6743-6836 (2016).

[5]. Asakura, S. and F. Oosawa, "On Interaction between Two Bodies Immersed in a Solution of Macromolecules," The Journal of Chemical Physics 22, 1255-1256 (1954).

[6]. Pham, K. N., A. M. Puertas, J. Bergenholtz, S. U. Egelhaaf, A. Moussaïd, P. N. Pusey, A. B. Schofield, M. E. Cates, M. Fuchs and W. C. K. Poon, "Multiple Glassy States in a Simple Model System," Science 296, 104-106 (2002).

[7]. Eckert, T. and E. Bartsch, "Re-entrant Glass Transition in a Colloid-Polymer Mixture with Depletion Attractions," Physical Review Letters 89, 125701 (2002).

[8]. Pham, K. N., S. U. Egelhaaf, P. N. Pusey and W. C. K. Poon, "Glasses in hard spheres with shortrange attraction," Physical Review E 69, 011503 (2004).

[9]. Lekkerkerker, H. N. W. and R. Tuinier, Colloids and the depletion interaction (Springer, Dordrecht, 2011), 833.

[10]. Pham, K. N., G. Petekidis, D. Vlassopoulos, S. U. Egelhaaf, W. C. K. Poon and P. N. Pusey, "Yielding behavior of repulsion- and attraction-dominated colloidal glasses," Journal of Rheology 52, 649-676 (2008).

[11]. Koumakis, N. and G. Petekidis, "Two step yielding in attractive colloids: transition from gels to attractive glasses," Soft Matter 7, 2456-2470 (2011).

[12]. Eckert, T. and E. Bartsch, "The effect of free polymer on the interactions and the glass transition dynamics of microgel colloids," Faraday Discussions 123, 51-64 (2003).

[13]. Laurati, M., S. U. Egelhaaf and G. Petekidis, "Plastic rearrangements in colloidal gels investigated by LAOS and LS-Echo," Journal of Rheology 58, 1395-1417 (2014). 
[14]. Crassous, J. J., L. Casal-Dujat, M. Medebach, M. Obiols-Rabasa, R. Vincent, F. Reinhold, V. Boyko, I. Willerich, A. Menzel, C. Moitzi, B. Reck and P. Schurtenberger, "Structure and Dynamics of Soft Repulsive Colloidal Suspensions in the Vicinity of the Glass Transition," Langmuir 29, 1034610359 (2013).

[15]. Wiemann, M., N. Willenbacher and E. Bartsch, "Effect of cross-link density on re-entrant melting of microgel colloids," Colloids and Surfaces A: Physicochemical and Engineering Aspects 413, 78-83 (2012).

[16]. Vlassopoulos, D. and M. Cloitre, "Tunable rheology of dense soft deformable colloids," Current opinion in colloid \& interface science 19, 561-574 (2014).

[17]. Yuan, G., H. Cheng and C. C. Han, "The glass formation of a repulsive system with also a short range attractive potential: A re-interpretation of the free volume theory," Polymer.

[18]. Wilk, A., S. Huißmann, E. Stiakakis, J. Kohlbrecher, D. Vlassopoulos, C. N. Likos, G. Meier, J. K. G. Dhont, G. Petekidis and R. Vavrin, "Osmotic shrinkage in star/linear polymer mixtures," Eur. Phys. J. E 32, 127-134 (2010).

[19]. Truzzolillo, D., D. Vlassopoulos and M. Gauthier, "Osmotic Interactions, Rheology, and Arrested Phase Separation of Star-Linear Polymer Mixtures," Macromolecules 44, 5043-5052 (2011).

[20]. Truzzolillo, D., D. Vlassopoulos, M. Gauthier and A. Munam, "Thermal melting in depletion gels of hairy nanoparticles," Soft Matter 9, 9088-9093 (2013).

[21]. Stiakakis, E., D. Vlassopoulos, C. N. Likos, J. Roovers and G. Meier, "Polymer-Mediated Melting in Ultrasoft Colloidal Gels," Physical Review Letters 89, 208302 (2002).

[22]. Stiakakis, E., G. Petekidis, D. Vlassopoulos, C. N. Likos, H. Iatrou, N. Hadjichristidis and J. Roovers, "Depletion and cluster formation in soft colloid - polymer mixtures," EPL (Europhysics Letters) 72, 664 (2005).

[23]. Lonetti, B., M. Camargo, J. Stellbrink, C. N. Likos, E. Zaccarelli, L. Willner, P. Lindner and D. Richter, "Ultrasoft Colloid-Polymer Mixtures: Structure and Phase Diagram," Physical Review Letters 106, 228301 (2011).

[24]. Truzzolillo, D., D. Vlassopoulos and M. Gauthier, "Rheological detection of caging and solidliquid transitions in soft colloid-polymer mixtures," Journal of Non-Newtonian Fluid Mechanics 193, 11-20 (2013).

[25]. Kandar, A. K., J. K. Basu, S. Narayanan and A. Sandy, "Anomalous structural and dynamical phase transitions of soft colloidal binary mixtures," Soft Matter 8, 10055-10060 (2012). 
[26]. Abbas, S. and T. P. Lodge, "Depletion Interactions: Effects of Added Homopolymer on Ordered Phases Formed by Spherical Block Copolymer Micelles," Macromolecules 41, 8895-8902 (2008).

[27]. Watanabe, H. and T. Kotaka, "Rheology of Ternary Mixtures of Styrene-Butadiene Diblock Copolymer, Homopolybutadiene, and n-Tetradecane," Journal of Rheology 27, 223-240 (1983).

[28]. Di Lorenzo, F. and S. Seiffert, "Counter-effect of Brownian and elastic forces on the liquid-tosolid transition of microgel suspensions," Soft Matter 11, 5235-5245 (2015).

[29]. Willenbacher, N., J. S. Vesaratchanon, O. Thorwarth and E. Bartsch, "An alternative route to highly concentrated, freely flowing colloidal dispersions," Soft Matter 7, 5777-5788 (2011).

[30]. Monti, F., S.-Y. Fu, I. Iliopoulos and M. Cloitre, "Doubly Responsive Polymer-Microgel Composites: Rheology and Structure," Langmuir 24, 11474-11482 (2008).

[31]. Saunders, B. R. and B. Vincent, "Osmotic de-swelling of polystyrene microgel particles," Colloid and Polymer Science 275, 9-17 (1997).

[32]. Fernández-Nieves, A., A. Fernández-Barbero, B. Vincent and F. J. de las Nieves, "Osmotic deswelling of ionic microgel particles," The Journal of Chemical Physics 119, 10383-10388 (2003).

[33]. Routh, A. F., A. Fernandez-Nieves, M. Bradley and B. Vincent, "Effect of Added Free Polymer on the Swelling of Neutral Microgel Particles: A Thermodynamic Approach," The Journal of Physical Chemistry B 110, 12721-12727 (2006).

[34]. Helgeson, M. E., S. C. Chapin and P. S. Doyle, "Hydrogel microparticles from lithographic processes: Novel materials for fundamental and applied colloid science," Current opinion in colloid \& interface science 16, 106-117 (2011).

[35]. Helgeson, M. E., Y. Gao, S. E. Moran, J. Lee, M. Godfrin, A. Tripathi, A. Bose and P. S. Doyle, "Homogeneous percolation versus arrested phase separation in attractively-driven nanoemulsion colloidal gels," Soft Matter 10, 3122-3133 (2014).

[36]. Helgeson, M. E., "Colloidal behavior of nanoemulsions: Interactions, structure, and rheology," Current opinion in colloid \& interface science 25, 39-50 (2016).

[37]. Mezzenga, R. and P. Fischer, "The self-assembly, aggregation and phase transitions of food protein systems in one, two and three dimensions," Reports on Progress in Physics 76, 046601 (2013).

[38]. Imhof, A. and J. K. G. Dhont, "Experimental Phase Diagram of a Binary Colloidal Hard-Sphere Mixture with a Large Size Ratio," Physical Review Letters 75, 1662-1665 (1995). 
[39]. Dinsmore, A. D., A. G. Yodh and D. J. Pine, "Phase diagrams of nearly-hard-sphere binary colloids," Physical Review E 52, 4045-4057 (1995).

[40]. Voigtmann, T., "Multiple glasses in asymmetric binary hard spheres," EPL (Europhysics Letters) 96, 36006 (2011).

[41]. Sentjabrskaja, T., M. Hermes, W. Poon, C. Estrada, R. Castaneda-Priego, S. Egelhaaf and M. Laurati, "Transient dynamics during stress overshoots in binary colloidal glasses," Soft Matter 10, 65466555 (2014).

[42]. Sentjabrskaja, T., E. Babaliari, J. Hendricks, M. Laurati, G. Petekidis and S. U. Egelhaaf, "Yielding of binary colloidal glasses," Soft Matter 9, 4524-4533 (2013).

[43]. Zaccarelli, E., C. Mayer, A. Asteriadi, C. N. Likos, F. Sciortino, J. Roovers, H. Iatrou, N. Hadjichristidis, P. Tartaglia, H. Löwen and D. Vlassopoulos, "Tailoring the Flow of Soft Glasses by Soft Additives," Physical Review Letters 95, 268301 (2005).

[44]. Mayer, C., E. Stiakakis, E. Zaccarelli, C. N. Likos, F. Sciortino, P. Tartaglia, H. Löwen and D. Vlassopoulos, "Rheological transitions in asymmetric colloidal star mixtures," Rheologica Acta 46, 611619 (2007).

[45]. Mayer, C., E. Zaccarelli, E. Stiakakis, C. N. Likos, F. Sciortino, A. Munam, M. Gauthier, N. Hadjichristidis, H. Iatrou, P. Tartaglia, H. Lowen and D. Vlassopoulos, "Asymmetric caging in soft colloidal mixtures," Nat Mater 7, 780-784 (2008).

[46]. Stiakakis, E., B. M. Erwin, D. Vlassopoulos, M. Cloitre, A. Munam, M. Gauthier, H. Iatrou and N. Hadjichristidis, "Probing glassy states in binary mixtures of soft interpenetrable colloids," Journal of Physics: Condensed Matter 23, 234116 (2011).

[47]. Bayliss, K., J. Van Duijneveldt, M. Faers and A. Vermeer, "Comparing colloidal phase separation induced by linear polymer and by microgel particles," Soft Matter 7, 10345-10352 (2011).

[48]. Luo, J., G. Yuan, C. Zhao, C. C. Han, J. Chen and Y. Liu, "Gelation of large hard particles with short-range attraction induced by bridging of small soft microgels," Soft Matter 11, 2494-2503 (2015).

[49]. Agrawal, A., H.-Y. Yu, S. Srivastava, S. Choudhury, S. Narayanan and L. A. Archer, "Dynamics and yielding of binary self-suspended nanoparticle fluids," Soft Matter 11, 5224-5234 (2015).

[50]. Chremos, A., A. Z. Panagiotopoulos and D. L. Koch, "Dynamics of solvent-free grafted nanoparticles," The Journal of Chemical Physics 136, 044902 (2012). 
[51]. Kim, S. A., R. Mangal and L. A. Archer, "Relaxation dynamics of nanoparticle-tethered polymer chains," Macromolecules 48, 6280-6293 (2015).

[52]. Puaud, F., E. Nicol, G. Brotons, T. Nicolai and L. Benyahia, "Liquid-Solid Transition and Crystallization of Mixtures of Frozen and Dynamic Star-Like Polymers," Macromolecules 47, 1175 1180 (2014).

[53]. Zhou, Z., D. Jia, J. V. Hollingsworth, H. Cheng and C. C. Han, "From repulsive to attractive glass: A rheological investigation," The Journal of Chemical Physics 143, 234901 (2015).

[54]. Eberle, A. P., N. J. Wagner and R. Castañeda-Priego, "Dynamical arrest transition in nanoparticle dispersions with short-range interactions," Physical Review Letters 106, 105704 (2011).

[55]. Narayanan, T., M. Sztucki, G. Belina and F. Pignon, "Microstructure and rheology near an attractive colloidal glass transition," Physical Review Letters 96, 258301 (2006).

[56]. Jia, D., J. V. Hollingsworth, Z. Zhou, H. Cheng and C. C. Han, "Coupling of gelation and glass transition in a biphasic colloidal mixture-from gel-to-defective gel-to-glass," Soft Matter 11, 88188826 (2015).

[57]. Zong, Y., G. Yuan, C. Zhao and C. C. Han, "Differentiating bonding and caging in a charged colloid system through rheological measurements," The Journal of Chemical Physics 138, 184902 (2013).

[58]. Zhou, Z., J. V. Hollingsworth, S. Hong, H. Cheng and C. C. Han, "Yielding behavior in colloidal glasses: comparison between "hard cage" and "soft cage"," Langmuir 30, 5739-5746 (2014).

[59]. Marzi, D., C. N. Likos and B. Capone, "Coarse graining of star-polymer - colloid nanocomposites," The Journal of Chemical Physics 137, 014902 (2012).

[60]. Mahynski, N. A. and A. Z. Panagiotopoulos, "Phase behavior of athermal colloid-star polymer mixtures," The Journal of Chemical Physics 139, 024907 (2013).

[61]. Truzzolillo, D., D. Marzi, J. Marakis, B. Capone, M. Camargo, A. Munam, F. Moingeon, M. Gauthier, C. N. Likos and D. Vlassopoulos, "Glassy States in Asymmetric Mixtures of Soft and Hard Colloids," Physical Review Letters 111, 208301 (2013).

[62]. Marzi, D., B. Capone, J. Marakis, M. C. Merola, D. Truzzolillo, L. Cipelletti, F. Moingeon, M. Gauthier, D. Vlassopoulos, C. N. Likos and M. Camargo, "Depletion, melting and reentrant solidification in mixtures of soft and hard colloids," Soft Matter 11, 8296-8312 (2015). 
[63]. Gauthier, M. and A. Munam, "Synthesis of 1,4-Polybutadiene Dendrimer-Arborescent Polymer Hybrids," Macromolecules 43, 3672-3681 (2010).

[64]. Daoud, M. and J. P. Cotton, "Star shaped polymers : a model for the conformation and its concentration dependence," J. Phys. France 43, 531-538 (1982).

[65]. Erwin, B. M., D. Vlassopoulos and M. Cloitre, "Rheological fingerprinting of an aging soft colloidal glass," Journal of Rheology 54, 915-939 (2010).

[66]. Helgeson, M. E., N. J. Wagner and D. Vlassopoulos, "Viscoelasticity and shear melting of colloidal star polymer glasses," Journal of Rheology 51, 297-316 (2007).

[67]. McLeish, T., "Tube theory of entangled polymer dynamics," Advances in physics 51, 1379-1527 (2002).

[68]. Lu, Y., X. Lu, Z. Qin and J. Shen, "Direct visualization of free-volume-triggered activation of $\beta$ relaxation in colloidal glass," Physical Review E 94, 012606 (2016).

[69]. Likos, C. N., H. Löwen, A. Poppe, L. Willner, J. Roovers, B. Cubitt and D. Richter, "Ordering phenomena of star polymer solutions approaching the $\Theta$ state," Physical Review E 58, 6299-6307 (1998).

[70]. Guth, E. and O. Gold, "On the hydrodynamical theory of the viscosity of suspensions," Phys. Rev. 53, (1938).

[71]. Truzzolillo, D., D. Vlassopoulos, A. Munam and M. Gauthier, "Depletion gels from dense soft colloids: Rheology and thermoreversible melting," Journal of Rheology 58, 1441-1462 (2014).

[72]. Koumakis, N., A. Pamvouxoglou, A. S. Poulos and G. Petekidis, "Direct comparison of the rheology of model hard and soft particle glasses," Soft Matter 8, 4271-4284 (2012).

[73]. Le Grand, A. and G. Petekidis, "Effects of particle softness on the rheology and yielding of colloidal glasses," Rheologica Acta 47, 579-590 (2008).

[74]. Erwin, B. M., M. Cloitre, M. Gauthier and D. Vlassopoulos, "Dynamics and rheology of colloidal star polymers," Soft Matter 6, 2825-2833 (2010).

[75]. Pellet, C. and M. Cloitre, "The glass and jamming transitions of soft polyelectrolyte microgel suspensions," Soft Matter 12, 3710-3720 (2016). 
[76]. Di Lorenzo, F. and S. Seiffert, "Particulate and continuum mechanics of microgel pastes: effect and non-effect of compositional heterogeneity," Colloid and Polymer Science 291, 2927-2933 (2013).

[77]. Kramb, R. C., R. Zhang, K. S. Schweizer and C. F. Zukoski, "Re-entrant kinetic arrest and elasticity of concentrated suspensions of spherical and nonspherical repulsive and attractive colloids," The Journal of Chemical Physics 134, 014503 (2011).

[78]. Kramb, R. C. and C. F. Zukoski, "Nonlinear rheology and yielding in dense suspensions of hard anisotropic colloids," Journal of Rheology 55, 1069-1084 (2011).

[79]. Chellamuthu, M., E. M. Arndt and J. P. Rothstein, "Extensional rheology of shear-thickening nanoparticle suspensions," Soft Matter 5, 2117-2124 (2009).

[80]. Lee, Y. S. and N. J. Wagner, "Dynamic properties of shear thickening colloidal suspensions," Rheologica Acta 42, 199-208 (2003).

[81]. Ballauff, M., J. M. Brader, S. U. Egelhaaf, M. Fuchs, J. Horbach, N. Koumakis, M. Krüger, M. Laurati, K. J. Mutch, G. Petekidis, M. Siebenbürger, T. Voigtmann and J. Zausch, "Residual Stresses in Glasses," Physical Review Letters 110, 215701 (2013).

[82]. Mohan, L., M. Cloitre and R. T. Bonnecaze, "Build-up and two-step relaxation of internal stress in jammed suspensions," Journal of Rheology 59, 63-84 (2015).

[83]. Mohan, L., R. T. Bonnecaze and M. Cloitre, "Microscopic Origin of Internal Stresses in Jammed Soft Particle Suspensions," Physical Review Letters 111, 268301 (2013).

[84]. Fritschi, S., M. Fuchs and T. Voigtmann, "Mode-coupling analysis of residual stresses in colloidal glasses," Soft Matter 10, 4822-4832 (2014).

[85]. Amann, C. P., M. Siebenbürger, M. Ballauff and M. Fuchs, "Nonlinear rheology of glass-forming colloidal dispersions: transient stress-strain relations from anisotropic mode coupling theory and thermosensitive microgels," Journal of Physics: Condensed Matter 27, 194121 (2015).

[86]. Ewoldt, R. H. and G. H. McKinley, "On secondary loops in LAOS via self-intersection of Lissajous-Bowditch curves," Rheologica Acta 49, 213-219 (2010).

[87]. Rogers, S. A., B. M. Erwin, D. Vlassopoulos and M. Cloitre, "A sequence of physical processes determined and quantified in LAOS: Application to a yield stress fluid," Journal of Rheology 55, 435458 (2011). 
[88]. Mohan, L., C. Pellet, M. Cloitre and R. Bonnecaze, "Local mobility and microstructure in periodically sheared soft particle glasses and their connection to macroscopic rheology," Journal of Rheology 57, 1023-1046 (2013).

[89]. Ewoldt, R. H., A. E. Hosoi and G. H. McKinley, "New measures for characterizing nonlinear viscoelasticity in large amplitude oscillatory shear," Journal of Rheology 52, 1427-1458 (2008).

[90]. Cho, K. S., K. Hyun, K. H. Ahn and S. J. Lee, "A geometrical interpretation of large amplitude oscillatory shear response," Journal of Rheology (1978-present) 49, 747-758 (2005).

[91]. Koumakis, N., J. F. Brady and G. Petekidis, "Complex Oscillatory Yielding of Model Hard-Sphere Glasses," Physical Review Letters 110, 178301 (2013).

[92]. Carotenuto, C., M. Grosso and P. L. Maffettone, "Fourier Transform Rheology of Dilute Immiscible Polymer Blends: A Novel Procedure To Probe Blend Morphology," Macromolecules 41, 4492-4500 (2008).

[93]. Ahirwal, D., S. Filipe, I. Neuhaus, M. Busch, G. Schlatter and M. Wilhelm, "Large amplitude oscillatory shear and uniaxial extensional rheology of blends from linear and long-chain branched polyethylene and polypropylene," Journal of Rheology 58, 635-658 (2014).

[94]. Kim, J., D. Merger, M. Wilhelm and M. E. Helgeson, "Microstructure and nonlinear signatures of yielding in a heterogeneous colloidal gel under large amplitude oscillatory shear," Journal of Rheology (1978-present) 58, 1359-1390 (2014).

[95]. Hyun, K. and M. Wilhelm, "Establishing a New Mechanical Nonlinear Coefficient Q from FTRheology: First Investigation of Entangled Linear and Comb Polymer Model Systems," Macromolecules 42, 411-422 (2009).

[96]. Reinheimer, K., M. Grosso, F. Hetzel, J. Kübel and M. Wilhelm, "Fourier Transform Rheology as an innovative morphological characterization technique for the emulsion volume average radius and its distribution," Journal of Colloid and Interface Science 380, 201-212 (2012).

[97]. Kate Gurnon, A. and N. J. Wagner, "Large amplitude oscillatory shear (LAOS) measurements to obtain constitutive equation model parameters: Giesekus model of banding and nonbanding wormlike micelles," Journal of Rheology 56, 333-351 (2012).

[98]. Hyun, K., W. Kim, S. Joon Park and M. Wilhelm, "Numerical simulation results of the nonlinear coefficient Q from FT-Rheology using a single mode pom-pom model," Journal of Rheology 57, 1-25 (2013). 
[99]. Wagner, M. H., V. H. Rolón-Garrido, K. Hyun and M. Wilhelm, "Analysis of medium amplitude oscillatory shear data of entangled linear and model comb polymers," Journal of Rheology 55, 495-516 (2011).

$[100]$.

[101]. Vittorias, I., D. Lilge, V. Baroso and M. Wilhelm, "Linear and non-linear rheology of linear polydisperse polyethylene," Rheologica Acta 50, 691-700 (2011).

[102]. Cziep, M. A., M. Abbasi, M. Heck, L. Arens and M. Wilhelm, "Effect of Molecular Weight, Polydispersity, and Monomer of Linear Homopolymer Melts on the Intrinsic Mechanical Nonlinearity 3Q0 $(\omega)$ in MAOS," Macromolecules 49, 3566-3579 (2016).

[103]. Pearson, D. S. and W. E. Rochefort, "Behavior of concentrated polystyrene solutions in largeamplitude oscillating shear fields," Journal of Polymer Science: Polymer Physics Edition 20, 83-98 (1982).

[104]. Brader, J. M., M. Siebenbürger, M. Ballauff, K. Reinheimer, M. Wilhelm, S. J. Frey, F. Weysser and M. Fuchs, "Nonlinear response of dense colloidal suspensions under oscillatory shear: Modecoupling theory and Fourier transform rheology experiments," Physical Review E 82, 061401 (2010). 